\title{
3D structure recovery and unwarping of surfaces applicable to planes
}

\author{
Nail A. Gumerov, Ali Zandifar, Ramani Duraiswami, and Larry S. Davis \\ Perceptual Interfaces and Reality Lab, University of Maryland, College Park, MD 20742
}

April 8, 2005

\begin{abstract}
The deformation of applicable surfaces such as sheets of paper satisfies the differential geometric constraints of isometry (lengths and areas are conserved) and vanishing Gaussian curvature. We show that these constraints lead to a closed set of equations that allow recovery of the full geometric structure from a single image of the surface and knowledge of its undeformed shape. We show that these partial differential equations can be reduced to the Hopf equation that arises in non-linear wave propagation, and deformations of the paper can be interpreted in terms of the characteristics of this equation. A new exact integration of these equations is developed that relates the 3-D structure of the applicable surface to an image. The solution is tested by comparison with particular exact solutions. We present results for both the forward and the inverse 3D structure recovery problem.
\end{abstract}

Keywords: 3D structure recovery, unwarping, applicable surface, differential geometry, single view

\section{Introduction}

When a picture or text printed on paper is imaged, we are presented with a problem of unwarping the captured digital image to its flat, fronto-parallel representation, as a preprocessing step before performing tasks such as identification, or Optical Character Recognition (OCR). In the case that the paper is flat, the problem reduces to one of undoing a projection of an initial shape such as a rectangle, and the rectification (or unwarping) can be achieved by computing a simple homography. A harder problem is when the piece of paper is itself deformed or bent. In this case the unwarping must undo both the effects of the three-dimensional bending of the surface, and the imaging process.

The differential geometry of surfaces provides a very powerful set of relations for analysis of the unwarping. However, most quantitative use of differential geometry has been restricted to range data, while its use for image data has been primarily qualitative. The deformation of paper surfaces satisfies the conditions of isometry and vanishing Gaussian curvature. Here, we show that these conditions can be analytically integrated to infer the complete 3D structure of the surface from an image of its bounding contour.

Previous authors have attempted to enforce these conditions in 3D reconstruction. However, they essentially enforced these as constraints to a process of polynomial/spline fitting using data 
obtained on the surface [17]. In contrast, we solve these equations, and show that information on the bounding contour is sufficient to determine structure completely. Further, exact correspondence information along the bounding contour is not needed. We only need the correspondences of a few points, e.g., corners. Other than its theoretical importance, our research can potentially benefit diverse computer vision applications, e.g. portable scanning devices, digital flattening of creased documents, 3D reconstruction without correspondence, and perhaps most importantly, OCR of scene text.

\section{Previous work}

A seminal paper by Koenderink [9] addressed the understanding of 3D structure qualitatively from occluding contours in images. It was shown that the concavities and convexities of visual contours are sufficient to infer the local shape of a surface. Here, we perform quantitative recovery of $3 \mathrm{D}$ surface structure for the case of applicable surfaces. While we were not able to find similar papers dealing with analytical integration of the equations of differential geometry to obtain structure, the following papers deal with related problems of unwarping scene text, or using differential geometric constraints for reconstruction.

Metric rectification of planar surfaces: In $[2,12,16]$ algorithms for performing metric rectification of planar surfaces were considered. These papers extract from the images, features such as vanishing lines and right angles and perform rectification. Extraction of vanishing lines is achieved by different methods; such as the projection profile method [2] and the illusory and non-illusory lines in textual layouts [16].

Undoing paper curl for non-planar surfaces knowing range data: A number of papers deal with correcting the curl of documents using known shape (e.g. cylinders) [7, 21]. These approaches all need 3D points on the surface to solve for the inverse mapping. In [17] sparse 3D data on the curled paper surface was obtained from a laser device. An approximate algorithm to fit an applicable surface through these points was developed that allowed obtaining dense depth data. The isometry constraint was approximately enforced by requiring that distances between adjacent nodes be constant. In [1] a mass-spring particle system framework was used for digital flattening of damaged documents using depth measurements, though the differential geometry constraints are not enforced.

Isometric mapping: In [8] an algorithm is developed to bend virtual paper without shearing or tearing. Ref. [15] considers the shape-from-motion problem for shapes deformed under isometric mapping.

\section{Theory}

\subsection{Basic surface representation}

In a 3D world coordinate system $(x, y, z)$, a surface $\mathbf{r}=(x, y, z)$, can be mathematically represented in explicit, implicit and parametric forms respectively as:

$$
z=f(x, y), \quad F(x, y, z)=0, \quad \mathbf{r}(u, v)=(X(u, v), Y(u, v), Z(u, v)) .
$$




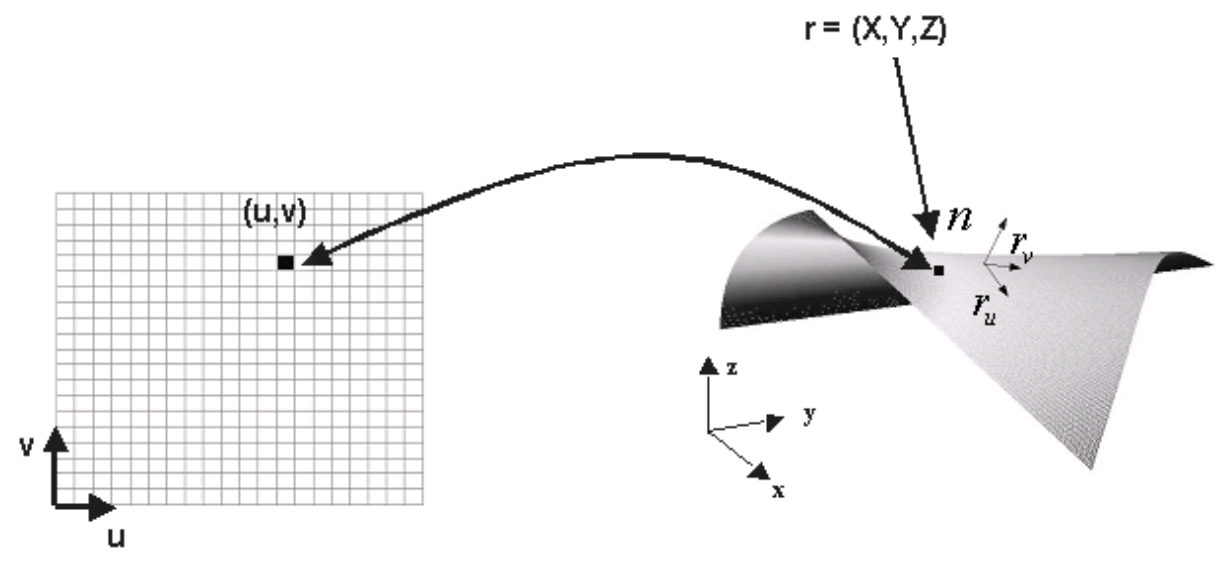

Figure 1: Parametric representation of a surface.

We consider a smooth surface $S$ expressed parametrically as $\mathbf{r}(u, v)$ in Equation (1), which is a mapping from any point $(u, v)$ in the parametric (or undeformed) plane, the $u v$-plane, to a point $(X, Y, Z)$ on the surface in $3 \mathrm{D}$ (see Figure 1).

The sets $\{\mathbf{r}(u, v), v=$ const $\}$ and $\{\mathbf{r}(u, v), u=$ const $\}$ represent two families of curves on the surface. The partial derivatives with respect to $u$ and $v$ can be denoted as $\mathbf{r}_{u}$ and $\mathbf{r}_{v}$ and are the tangent vectors to the curves $v=$ const and $u=$ const, respectively [11]. The element of distance $d s=|d \mathbf{r}|$ on the surface is given at each surface point $(u, v)$ by the first fundamental form of a surface

$$
\begin{aligned}
& d s^{2}=|d \mathbf{r}|^{2}=\left|\mathbf{r}_{u}\right|^{2} d u^{2}+2 \mathbf{r}_{u} \cdot \mathbf{r}_{v} d u d v+\left|\mathbf{r}_{v}\right|^{2} d v^{2}=E d u^{2}+2 F d u d v+G d v^{2}, \\
& E(u, v)=\left|\mathbf{r}_{u}\right|^{2}, \quad F(u, v)=\mathbf{r}_{u} \cdot \mathbf{r}_{v}, \quad G(u, v)=\left|\mathbf{r}_{v}\right|^{2} .
\end{aligned}
$$

The surface coordinates are orthogonal iff $F \equiv 0$. The surface normal $\mathbf{n}$ and area element $d A$ can be defined in terms of the tangent vectors as:

$$
\mathbf{n}=\frac{\mathbf{r}_{u} \times \mathbf{r}_{v}}{\left|\mathbf{r}_{u} \times \mathbf{r}_{v}\right|}, \quad d A=\left|\mathbf{r}_{u} \times \mathbf{r}_{v}\right| d u d v=\sqrt{E G-F^{2}} d u d v .
$$

The second fundamental form of a surface at a point $(u, v)$ measures how far the surface is from being planar. It is given by

$$
-d \mathbf{r} \cdot d \mathbf{n}=L(u, v) d u^{2}+2 M(u, v) d u d v+N(u, v) d v^{2},
$$

where $L, M$ and $N$ are defined as[11]:

$$
\begin{aligned}
L(u, v) & =-\mathbf{r}_{u} \cdot \mathbf{n}_{u}=\mathbf{r}_{u u} \cdot \mathbf{n}, \\
M(u, v) & =-\mathbf{r}_{u} \cdot \mathbf{n}_{v}=\mathbf{r}_{u v} \cdot \mathbf{n}, \\
N(u, v) & =-\mathbf{r}_{v} \cdot \mathbf{n}_{v}=\mathbf{r}_{v v} \cdot \mathbf{n} .
\end{aligned}
$$


Here the second derivatives of $\mathbf{r}$ with respect to $u$ and $v$ are denoted as $\mathbf{r}_{u u}, \mathbf{r}_{u v}$ and $\mathbf{r}_{v v}$.

For every normal section through $(u, v)$ there exist two principal curvatures $k_{1}$ and $k_{2}$. The mean, $H(u, v)$, and the Gaussian, $K(u, v)$, curvatures are defined as:

$$
H \equiv \frac{k_{1}+k_{2}}{2}=\frac{1}{2} \frac{E N-2 F M+G L}{E G-F^{2}}, \quad K \equiv k_{1} k_{2}=\frac{L N-M^{2}}{E G-F^{2}} .
$$

\subsection{Special surfaces}

Let us assume that we have a mapping of a point in the parametric plane $(u, v)$ to a point in $3 \mathrm{D}$ $\mathbf{r}=(X, Y, Z)$. The mapping is isometric if the length of a curve or element of area is invariant with the mapping, i.e.

$$
E(u, v)=\left|\mathbf{r}_{u}\right|^{2}=1, \quad F(u, v)=\mathbf{r}_{u} \cdot \mathbf{r}_{v}=0, \quad G(u, v)=\left|\mathbf{r}_{v}\right|^{2}=1 .
$$

Lengths and areas are conserved in an isometric mapping

$$
\begin{aligned}
& d s^{2}=|d \mathbf{r}|^{2}=E(u, v) d u^{2}+2 F(u, v) d u d v+G(u, v) d v^{2}=d u^{2}+d v^{2}, \\
& d A=\sqrt{E G-F^{2}} d u d v=d u d v .
\end{aligned}
$$

The mapping is conformal if the angle between curves on a surface is invariant of the mapping $(F=0)$. It is developable if the Gaussian curvature is zero everywhere.

$$
K=0 \Longrightarrow L N-M^{2}=0 .
$$

It is stated in [10] that two surfaces are applicable to each other if the mapping between them conserves the first fundamental form, and the Gaussian curvature. According to this definition a surface is applicable to a plane if the surface is isometric with a plane (7) and the Gaussian curvature vanishes (9) for every point on the surface. In this paper we simplify the terminology and refer to a surface applicable to a plane as an "applicable surface."

\section{Partial differential equations describing an applicable surface}

Here we prove two theorems, which provide partial differential equations that describe applicable surfaces.

Theorem 1 For a surface $\mathbf{r}=\mathbf{r}(u, v)$ isometric to a plane, the normal vector $\mathbf{n}$ is collinear to the vectors $\mathbf{r}_{u u}, \mathbf{r}_{u v}, \mathbf{r}_{v v}$ :

$\mathbf{n}\left\|\mathbf{r}_{u u}\right\| \mathbf{r}_{u v} \| \mathbf{r}_{v v}$.

Proof. If we differentiate the first and third equations in (7) with respect to $u$ and $v$, we obtain:

$$
\mathbf{r}_{u u} \cdot \mathbf{r}_{u}=\mathbf{r}_{u u} \cdot \mathbf{r}_{v}=\mathbf{r}_{u v} \cdot \mathbf{r}_{u}=\mathbf{r}_{u v} \cdot \mathbf{r}_{v}=\mathbf{r}_{v v} \cdot \mathbf{r}_{u}=\mathbf{r}_{v v} \cdot \mathbf{r}_{v}=0 .
$$


This shows that the vectors $\mathbf{r}_{u u}=\left(X_{u u}, Y_{u u}, Z_{u u}\right), \mathbf{r}_{u v}=\left(X_{u v}, Y_{u v}, Z_{u v}\right)$, and $\mathbf{r}_{v v}=\left(X_{v v}, Y_{v v}, Z_{v v}\right)$ are perpendicular both to $\mathbf{r}_{u}$ and $\mathbf{r}_{v}$ and, consequently, are collinear with the normal vector to the surface. This can be written as:

$$
\mathbf{n}=a \mathbf{r}_{u u}=b \mathbf{r}_{u v}=c \mathbf{r}_{v v}
$$

or in the form (10).

Corollary 2 Let $W(u, v)$ and $Q(u, v)$ denote any of the functions $X(u, v), Y(u, v)$, and $Z(u, v)$ which describe a surface isometric to a plane. Then

$$
W_{u u} Q_{v v}=W_{v v} Q_{u u}, \quad W_{u u} Q_{u v}=W_{u v} Q_{u u}, \quad W, Q=X, Y, Z .
$$

Proof. It follows from Theorem 1 that $\mathbf{r}_{u u} \times \mathbf{r}_{v v}=\mathbf{0}$ and $\mathbf{r}_{u u} \times \mathbf{r}_{u v}=\mathbf{0}$. These equations written for each component in Cartesian coordinates $\mathbf{r}(u, v)=(X(u, v), Y(u, v), Z(u, v))$ result in (13).

Theorem 3 Let $W(u, v)$ denote any of the functions $X(u, v), Y(u, v)$, and $Z(u, v)$ which describe an applicable surface. Then

$$
W_{u u} W_{v v}=W_{u v}^{2}, \quad W=X, Y, Z .
$$

Proof. Substituting (5) into (9) we obtain for surfaces of zero Gauss curvature:

$$
\left(\mathbf{r}_{u u} \cdot \mathbf{n}\right)\left(\mathbf{r}_{v v} \cdot \mathbf{n}\right)-\left(\mathbf{r}_{u v} \cdot \mathbf{n}\right)^{2}=0 .
$$

Then we can use the relation (12) for surfaces isometric to a plane. For applicable surfaces, then

$$
a c-b^{2}=0,
$$

where $a, b$, and $c$ are the scalars in (12). Further

$$
\frac{W_{u v}}{W_{u u}}=\frac{a}{b}=\frac{b}{c}=\frac{W_{v v}}{W_{u v}}, \quad W=X, Y, Z
$$

Solving the set of nonlinear higher order partial differential equations (PDEs) (14) one can compute the surface structure $\mathbf{r}$ in $3 \mathrm{D}$, given boundary conditions (the boundary curves) for an applicable surface. These equations may be solved by conventional methods of numerically solving PDEs e.g., using the Finite Difference method or the Finite Element Method. However, the development and implementation of efficient solvers for nonlinear PDE can be a difficult task. Below we provide a much more efficient method based on reduction of the order of PDEs and, finally, reduction of the problem to integration of several simultaneous ordinary differential equations (ODEs). 


\subsection{First integral and reduction to ordinary differential equations}

Below we formulate our main results, which demonstrates the integrability of the system of PDEs describing applicable surfaces. These theorems also provide explicit expressions for integrals and conditions on the characteristics of the PDEs that can be treated as ODEs, which further can be used for solution of forward and inverse problems for warping and unwarping.

Theorem 4 Let $\mathbf{r}=\mathbf{r}(u, v)$ be an applicable surface with tangent vectors $\mathbf{r}_{u}(u, v)$ and $\mathbf{r}_{v}(u, v)$. Then there exists a scalar function (which we call the mapping function) $t=t(u, v)$, such that

$$
\mathbf{r}_{u}(u, v)=\mathbf{R}^{(u)}(t(u, v)), \quad \mathbf{r}_{v}=\mathbf{R}^{(v)}(t(u, v)),
$$

where $\mathbf{R}^{(u)}(t)$ and $\mathbf{R}^{(v)}(t)$ are some vector functions of the single variable $t$.

Proof. Consider the functions $X_{u}(u, v)$ and $X_{v}(u, v)$. These functions satisfy the following consistency conditions

$$
X_{u v}=\frac{\partial X_{u}}{\partial v}=\frac{\partial X_{v}}{\partial u}=X_{v u}
$$

With this condition, equation (14) for $W=X$ can be interpreted as the degeneracy condition for the Jacobian of the mapping $(u, v) \longmapsto\left(X_{u}, X_{v}\right)$ :

$$
\frac{\partial\left(X_{u}, X_{v}\right)}{\partial(u, v)}=\frac{\partial X_{u}}{\partial u} \frac{\partial X_{v}}{\partial v}-\frac{\partial X_{u}}{\partial v} \frac{\partial X_{v}}{\partial u}=0
$$

This shows that there exists a function $t^{(x)}(u, v)$, such that

$$
X_{u}(u, v)=\widetilde{X}^{(u)}\left(t^{(x)}(u, v)\right), \quad X_{v}(u, v)=\widetilde{X}^{(v)}\left(t^{(x)}(u, v)\right),
$$

where $\widetilde{X}^{(u)}\left(t^{(x)}\right)$ and $\widetilde{X}^{(v)}\left(t^{(x)}\right)$ are some scalar functions of the single variable $t^{(x)}$. Similarly, the consistency condition and equation (14) can be applied to the functions $Y_{u}, Y_{v}$ to show that there exists a function $t^{(y)}(u, v)$, such that these derivatives can be considered as functions of a single variable $t^{(y)}$ :

$$
Y_{u}(u, v)=\tilde{Y}^{(u)}\left(t^{(y)}(u, v)\right), \quad Y_{v}(u, v)=\tilde{Y}^{(v)}\left(t^{(y)}(u, v)\right) .
$$

Using the prime to denote derivatives of functions, we have from equations (21) and (22):

$$
X_{u u}=\widetilde{X}^{(u) \prime} \frac{\partial t^{(x)}}{\partial u}, \quad X_{u v}=\widetilde{X}^{(u) \prime} \frac{\partial t^{(x)}}{\partial v}, \quad Y_{u v}=\widetilde{Y}^{(u) \prime} \frac{\partial t^{(y)}}{\partial v}, \quad Y_{u u}=\widetilde{Y}^{(u) \prime} \frac{\partial t^{(y)}}{\partial u} .
$$

Now if we substitute these relations into the second equation (13), where we set $W=X$ and $Q=Y$, we can see that the Jacobian of the transform $(u, v) \longmapsto\left(t^{(x)}, t^{(y)}\right)$ is zero:

$$
\frac{\partial\left(t^{(x)}, t^{(y)}\right)}{\partial(u, v)}=\frac{\partial t^{(x)}}{\partial u} \frac{\partial t^{(y)}}{\partial v}-\frac{\partial t^{(x)}}{\partial v} \frac{\partial t^{(y)}}{\partial u}=0 .
$$


This means that there exists a function $t(u, v)$, such that

$$
t^{(x)}(u, v)=T^{(x)}(t(u, v)), \quad t^{(y)}(u, v)=T^{(y)}(t(u, v)),
$$

where $T^{(x)}(t)$ and $T^{(y)}(t)$ are functions of a single variable $t$. Therefore, if we define functions $X^{(u)}, X^{(v)}, Y^{(u)}$, and $Y^{(v)}$ of variable $t$ as

$$
\begin{array}{ll}
X^{(u)}(t)=\tilde{X}^{(u)}\left(T^{(x)}(t)\right), & X^{(v)}(t)=\tilde{X}^{(v)}\left(T^{(x)}(t)\right), \\
Y^{(u)}(t)=\tilde{Y}^{(u)}\left(T^{(y)}(t)\right), & Y^{(v)}(t)=\tilde{Y}^{(v)}\left(T^{(y)}(t)\right)
\end{array}
$$

we obtain

$$
X_{u}=X^{(u)}(t), \quad X_{v}=X^{(v)}(t), \quad Y_{u}=Y^{(u)}(t), \quad Y_{v}=Y^{(v)}(t) .
$$

Obviously, we can add to this list the relations

$$
Z_{u}=Z^{(u)}(t), \quad Z_{v}=Z^{(v)}(t),
$$

since all the above relations hold also for the $z$-component (one can simply replace all $Y$ 's with $Z$ 's). This proves the theorem, since the scalar functions can be arranged as components of a vector function as:

$$
\mathbf{R}^{(u)}(t)=\left(X^{(u)}(t), Y^{(u)}(t), Z^{(u)}(t)\right), \quad \mathbf{R}^{(v)}(t)=\left(X^{(v)}(t), Y^{(v)}(t), Z^{(v)}(t)\right) .
$$

Corollary 5 If the conditions of theorem 4 hold, the normal to the applicable surface is a function of t alone:

$$
\mathbf{n}(u, v)=\mathbf{N}(t(u, v)), \quad \mathbf{N}(t)=\mathbf{R}^{(u)}(t) \times \mathbf{R}^{(v)}(t) .
$$

Proof. This follows from the fact that for an applicable surface $\mathbf{n}=\mathbf{r}_{u} \times \mathbf{r}_{v}=\mathbf{R}^{(u)}(t(u, v)) \times$ $\mathbf{R}^{(v)}(t(u, v))$.

Theorem $\mathbf{6}$ Let $\mathbf{r}=\mathbf{r}(u, v)$ be an applicable surface with the mapping function $t(u, v)$, introduced by equation (18). Also let the vector $\mathbf{w}$ defined as

$$
\mathbf{w}=u \dot{\mathbf{R}}^{(u)}(t)+v \dot{\mathbf{R}}^{(v)}(t)
$$

be non-zero in some domain $(u, v) \in \Omega$. Then, in this domain $\mathbf{w}=\mathbf{w}(t)$ and the function $t(u, v)$ satisfies the Hopf equation

$$
h^{(v)}(t) \frac{\partial t}{\partial u}-h^{(u)}(t) \frac{\partial t}{\partial v}=0,
$$

where $h^{(v)}(t)$ and $h^{(u)}(t)$ are functions related to $\mathbf{R}^{(u)}(t)$ and $\mathbf{R}^{(v)}(t)$ as

$$
h^{(v)}(t) \dot{\mathbf{R}}^{(u)}(t)-h^{(u)}(t) \dot{\mathbf{R}}^{(v)}(t)=0, \quad \dot{\mathbf{R}}^{(u)}=\frac{d \mathbf{R}^{(u)}}{d t}, \quad \dot{\mathbf{R}}^{(v)}=\frac{d \mathbf{R}^{(v)}}{d t} .
$$

The solution $t(u, v)$ of equation (31) in implicit form is

$$
u h^{(u)}(t)+v h^{(v)}(t)=1 .
$$


Proof. Let us prove first that the function $\mathbf{w}$ introduced by (30) is a function of $t$ alone. Indeed, differentiating (18) we have

$$
\dot{\mathbf{R}}^{(u)} \frac{\partial t}{\partial v}=\mathbf{r}_{u v}=\mathbf{r}_{v u}=\dot{\mathbf{R}}^{(v)} \frac{\partial t}{\partial u} .
$$

This shows that the Jacobian of the transform $(u, v) \longmapsto(t, \mathbf{m} \cdot \mathbf{w})$, where $\mathbf{m}$ is an arbitrary constant vector, is zero:

$$
\begin{aligned}
& \frac{\partial(t, \mathbf{w} \cdot \mathbf{m})}{\partial(u, v)}=\frac{\partial t}{\partial u} \frac{\partial \mathbf{w} \cdot \mathbf{m}}{\partial v}-\frac{\partial t}{\partial v} \frac{\partial \mathbf{w} \cdot \mathbf{m}}{\partial u}=\frac{\partial t}{\partial u}\left[u \ddot{\mathbf{R}}^{(u)} \frac{\partial t}{\partial v}+\dot{\mathbf{R}}^{(v)}+v \ddot{\mathbf{R}}^{(v)} \frac{\partial t}{\partial v}\right] \cdot \mathbf{m} \\
& -\frac{\partial t}{\partial v}\left[\dot{\mathbf{R}}^{(u)}+u \ddot{\mathbf{R}}^{(u)} \frac{\partial t}{\partial u}+v \ddot{\mathbf{R}}^{(v)} \frac{\partial t}{\partial u}\right] \cdot \mathbf{m}=-\left(\dot{\mathbf{R}}^{(v)} \frac{\partial t}{\partial u}-\dot{\mathbf{R}}^{(u)} \frac{\partial t}{\partial v}\right) \cdot \mathbf{m}=0 .
\end{aligned}
$$

Setting here $\mathbf{m}=\mathbf{i}_{x}, \mathbf{i}_{y}, \mathbf{i}_{z}\left(\mathbf{i}_{x}, \mathbf{i}_{y}, \mathbf{i}_{z}\right.$ are the unit basis vectors) we can see that any component of vector $\mathbf{w}$ is a function of $t$ alone, and so $\mathbf{w}=\mathbf{w}(t)$.

Now we note that because the vectors

$$
\mathbf{r}_{u u}=\dot{\mathbf{R}}^{(u)} \frac{\partial t}{\partial u}, \quad \mathbf{r}_{v v}=\dot{\mathbf{R}}^{(v)} \frac{\partial t}{\partial v}
$$

are collinear (see (10)), the vectors $\dot{\mathbf{R}}^{(u)}$ and $\dot{\mathbf{R}}^{(v)}$ are collinear, and since $\mathbf{w}$ is a linear combination of these vectors (30), we have $\mathbf{w}(t)\left\|\dot{\mathbf{R}}^{(u)}(t)\right\| \dot{\mathbf{R}}^{(v)}(t)$. Hence, there exist scalar functions $h^{(u)}(t)$ and $h^{(v)}(t)$ such, that

$$
\dot{\mathbf{R}}^{(u)}(t)=h^{(u)}(t) \mathbf{w}(t), \quad \dot{\mathbf{R}}^{(v)}=h^{(v)}(t) \mathbf{w}(t) .
$$

Multiplying the first equation by $h^{(v)}(t)$ and subtracting the second equation multiplied by $h^{(u)}(t)$ we obtain relation (32). Substituting (37) into (34) we can see that $t(u, v)$ satisfies equation (31) because $\mathbf{w}$ is not zero. Equation (33) then can be obtained by substituting (37) into the definition (30).

Note that the Hopf equation is a common nonlinear hyperbolic equation in shock-wave theory, and its properties are well studied, see e.g. [20]. The characteristics of this equation, $t(u, v)=$ const, are straight lines in the $u v$-plane, as can be easily seen from equation (33). This also shows that the ratio $h^{(v)}\left(t_{*}\right) / h^{(u)}\left(t_{*}\right)$ describes the tangent of the characteristic $t(u, v)=t_{*}$. Along the characteristics all functions of $t$ are constant, and therefore the tangent vectors $\mathbf{r}_{u}(u, v)=\mathbf{R}^{(u)}(t)$ and $\mathbf{r}_{v}(u, v)=\mathbf{R}^{(v)}(t)$ do not change.

These facts allow us to perform integration of equations (18). This is provided by the following theorem.

Theorem 7 Let $\mathbf{r}=\mathbf{r}(u, v)$ be an applicable surface with the mapping function $t(u, v)$, introduced by equation (18) and let the vector $\mathbf{w}$ defined by relation (30) be non-zero in some domain $(u, v) \in$ $\Omega$. Then the solution of equations (18) in $\Omega$ is

$$
\mathbf{r}(u, v)=u \mathbf{R}^{(u)}(t)+v \mathbf{R}^{(v)}(t)+\boldsymbol{\rho}(t), \quad \dot{\boldsymbol{\rho}}(t)=-\mathbf{w}(t) .
$$




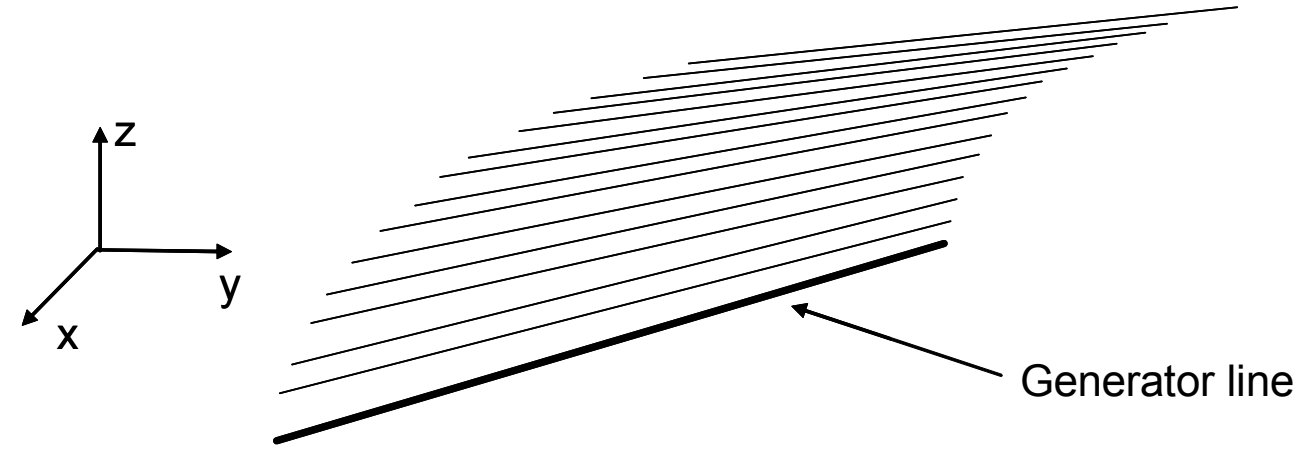

Figure 2: Generation of an applicable surface by sweeping a straight line in space.

Proof. To check that this is a solution, we can differentiate the first equation in (38) with respect to $u$ and $v$ and use the second equation in (38) and the definition of $\mathbf{w}(30)$. Doing this we find

$$
\begin{aligned}
\mathbf{r}_{u} & =\mathbf{R}^{(u)}(t)+\left[u \dot{\mathbf{R}}^{(u)}(t)+v \dot{\mathbf{R}}^{(v)}(t)+\dot{\boldsymbol{\rho}}(t)\right] \frac{\partial t}{\partial u} \\
& =\mathbf{R}^{(u)}(t)+\left[u \dot{\mathbf{R}}^{(u)}(t)+v \dot{\mathbf{R}}^{(v)}(t)-\mathbf{w}(t)\right] \frac{\partial t}{\partial u}=\mathbf{R}^{(u)}(t), \\
\mathbf{r}_{v} & =\mathbf{R}^{(v)}(t)+\left[u \dot{\mathbf{R}}^{(u)}(t)+v \dot{\mathbf{R}}^{(v)}(t)+\dot{\boldsymbol{\rho}}(t)\right] \frac{\partial t}{\partial v} \\
& =\mathbf{R}^{(v)}(t)+\left[u \dot{\mathbf{R}}^{(u)}(t)+v \dot{\mathbf{R}}^{(v)}(t)-\mathbf{w}(t)\right] \frac{\partial t}{\partial v}=\mathbf{R}^{(v)}(t) .
\end{aligned}
$$

Corollary 8 If the conditions of theorem 7 hold, the curves on the surface $\mathbf{r}=\mathbf{r}(u, v)$ corresponding to characteristics $t(u, v)=$ const. are straight lines.

Proof. Consider a characteristic line $t=t^{*}$. The tangent vectors $\mathbf{R}^{(u)}\left(t^{*}\right), \mathbf{R}^{(v)}\left(t^{*}\right)$ and vector $\boldsymbol{\rho}\left(t^{*}\right)$ from equation (38) are constant. If in equation (33) $h^{(v)}\left(t^{*}\right) \neq 0$ then along the characteristic line $v$ is a linear function of $u$ and

$$
\mathbf{r}=u \mathbf{R}^{(u)}\left(t^{*}\right)+\frac{\left(1-u h^{(u)}\left(t^{*}\right)\right)}{h^{(v)}\left(t^{*}\right)} \mathbf{R}^{(v)}\left(t^{*}\right)+\boldsymbol{\rho}\left(t^{*}\right)=u \mathbf{q}+\mathbf{q}_{0},
$$

where $\mathbf{q}_{0}$ and $\mathbf{q}$ are constant vectors. If $h^{(u)}\left(t^{*}\right) \neq 0$ then along the characteristic line

$$
\mathbf{r}=v \mathbf{R}^{(v)}\left(t^{*}\right)+\frac{\left(1-v h^{(v)}\left(t^{*}\right)\right)}{h^{(u)}\left(t^{*}\right)} \mathbf{R}^{(u)}\left(t^{*}\right)+\boldsymbol{\rho}\left(t^{*}\right)=v \mathbf{p}+\mathbf{p}_{0},
$$

where $\mathbf{q}_{0}$ and $\mathbf{q}$ are constant vectors. Recall that these are the equations of a line in 3D and according to equation (33) $h^{(u)}\left(t^{*}\right)$ and $h^{(v)}\left(t^{*}\right)$ cannot be zero simultaneously in the domain.

This corollary shows that one can sweep a straight line in space and the generated envelope will be an applicable surface (see Figure 2). It is also noteworthy that hyperbolic equations allow piecewise smooth solutions with discontinuities of the function $t(u, v)$ along the characteristics (shock 
waves). For the current problem, such solutions correspond to discontinuity of the tangent vectors to the surface along the characteristics, which are straight lines. There can also be a case when two different characteristics corresponding to, say, $t_{1}^{*}$ and $t_{2}^{*}$ intersect. In this situation, at the point of intersection we have two different values of tangent vectors, say $\mathbf{R}^{(u)}\left(t_{1}^{*}\right)$ and $\mathbf{R}^{(u)}\left(t_{2}^{*}\right)$. This means that the intersection point is the point of discontinuity in the solution. While consideration of the non-smooth solutions can be important for some problems, in the present paper we will concentrate only on the smooth bending of some patch (domain). In this case, for each point on the patch we can assign just one characteristic line and the solution is differentiable in the entire domain.

Note that there exists a special case when the vector $\mathbf{w}$ defined by equation (30) is zero in the domain under consideration. This special case includes the case when the surface is a plane, $\dot{\mathbf{R}}^{(u)}(t)=\dot{\mathbf{R}}^{(v)}(t)=\mathbf{0}$, and non-trivial cases, which are described by the Hopf-type equation with the family of characteristics passing through a single center $u=v=0$ (conical surfaces). In fact, the last case is not special when we consider warping and unwarping problems for a finite simplyconnective domain (patch) in the $(u, v)$-plane. They can be reduced to the case where $\mathbf{w} \neq \mathbf{0}$ by shifting the origin of the reference frame in the parametric plane in such a way that the point of characteristic intersection (singularity of the surface) is outside the domain.

The above equations are sufficient to solve the basic warping and unwarping problems for images based on information about the shapes of the image, or patch, boundaries. The goal is to find dependencies $\mathbf{R}^{(u)}(t), \mathbf{R}^{(v)}(t), \boldsymbol{\rho}(t), h^{(u)}(t)$ and $h^{(v)}(t)$ and, finally, $\mathbf{r}(u, v)$ from the available information. For convenience, we write down a summary of the differential and algebraic relations for applicable surfaces

$$
\begin{aligned}
& \mathbf{r}(u, v)=u \mathbf{R}^{(u)}(t)+v \mathbf{R}^{(v)}(t)+\boldsymbol{\rho}(t), \\
& \dot{\mathbf{R}}^{(u)}(t)=h^{(u)}(t) \mathbf{w}(t), \\
& \dot{\mathbf{R}}^{(v)}(t)=h^{(v)}(t) \mathbf{w}(t), \\
& \dot{\boldsymbol{\rho}}(t)=-\mathbf{w}(t), \\
& u h^{(u)}(t)+v h^{(v)}(t)=1, \\
& \left|\mathbf{R}^{(u)}\right|^{2}=1, \quad \mathbf{R}^{(u)} \cdot \mathbf{R}^{(v)}=0, \quad\left|\mathbf{R}^{(v)}\right|^{2}=1 .
\end{aligned}
$$

\section{Forward problem: generation of an applicable surface with a spec- ified boundary curve}

\subsection{Statement}

The first problem we consider is how to generate an applicable surface (or some patch on an applicable surface). We call this the forward problem. This problem is important, for example, for computer simulation of bending of a flat page in three dimensions. As we show below it is possible to do so by conforming some part of an initially flat patch (page edge) to a given curve in $3 \mathrm{D}$. In general the page edge can have an arbitrary given shape, while an important particular case is when the edge of the undeformed page is a straight line (see Fig. 3). We will consider both situations.

Consider a patch $\Omega^{\prime}$ in the $u v$-plane. Let $\Gamma^{\prime}$ be a curve describing some part of the patch edge, 
and let it be given parametrically as

$$
\Gamma^{\prime}: \quad u=U(t), \quad v=V(t), \quad u^{2}+v^{2} \neq 0, \quad t \in\left[t_{\min }, t_{\max }\right] .
$$

Without any loss of generality, we can assume that $t$ is a natural parameter of the curve, i.e. the distance along the curve measured from some starting point $A^{\prime}=\left(u_{0}, v_{0}\right) \in \Gamma^{\prime}$ :

$$
t=\int_{0}^{t} d s \equiv \int_{0}^{t} \sqrt{d \mathbf{r} \cdot d \mathbf{r}}
$$

For a natural parametrization, the functions $U(t)$ and $V(t)$ satisfy the additional constraint:

$$
\dot{U}^{2}+\dot{V}^{2}=1
$$

Assume that the page is warped and the patch $\Omega^{\prime}$ transforms to a patch $\Omega$, and the curve $\Gamma^{\prime}$ transforms to a curve $\Gamma$, whose parametric equation in $3 \mathrm{D}$ is

$$
\Gamma: \quad \mathbf{r}=\widetilde{\mathbf{R}}(\theta) .
$$

Due to the isometry of the transform, we can use the natural parameter $t$ instead of an arbitrary parameter $\theta$ to parametrize the warped curve $\Gamma$. The relation between the two parametrizations, then, is given by

$$
\mathbf{R}(t)=\widetilde{\mathbf{R}}(\theta), \quad \frac{d t}{d \theta}=\left|\frac{d \widetilde{\mathbf{R}}(\theta)}{d \theta}\right|,
$$

and we have

$$
\dot{\mathbf{R}} \cdot \dot{\mathbf{R}}=1 \text {. }
$$

\subsection{Equations}

Our goal is to write a set of equations that can be solved to determine all the unknown functions of $t$. We assume that all the assumptions that were made to derive equations (43) hold. Then, writing these equations for the values of $u$ and $v$ located on the boundary curve (44), we obtain:

$$
\begin{aligned}
& \mathbf{R}^{(u)} \cdot \mathbf{R}^{(u)}=1, \quad \mathbf{R}^{(u)} \cdot \mathbf{R}^{(v)}=0, \quad \mathbf{R}^{(v)} \cdot \mathbf{R}^{(v)}=1, \\
& U h^{(u)}+V h^{(v)}=1, \quad h^{(v)} \dot{\mathbf{R}}^{(u)}-h^{(u)} \dot{\mathbf{R}}^{(v)}=\mathbf{0}, \quad U \mathbf{R}^{(u)}+V \mathbf{R}^{(v)}+\boldsymbol{\rho}=\mathbf{R} .
\end{aligned}
$$

This system is completed by equations (46) and (49), which characterize the selection of $t$ as the natural parameter along the boundary curves. While the number of unknowns here is 11 $\left(\mathbf{R}^{(u)}, \mathbf{R}^{(v)}, \boldsymbol{\rho}, h^{(u)}, h^{(v)}\right)$ and the number of equations is 12 , two of these equations are dependent. 

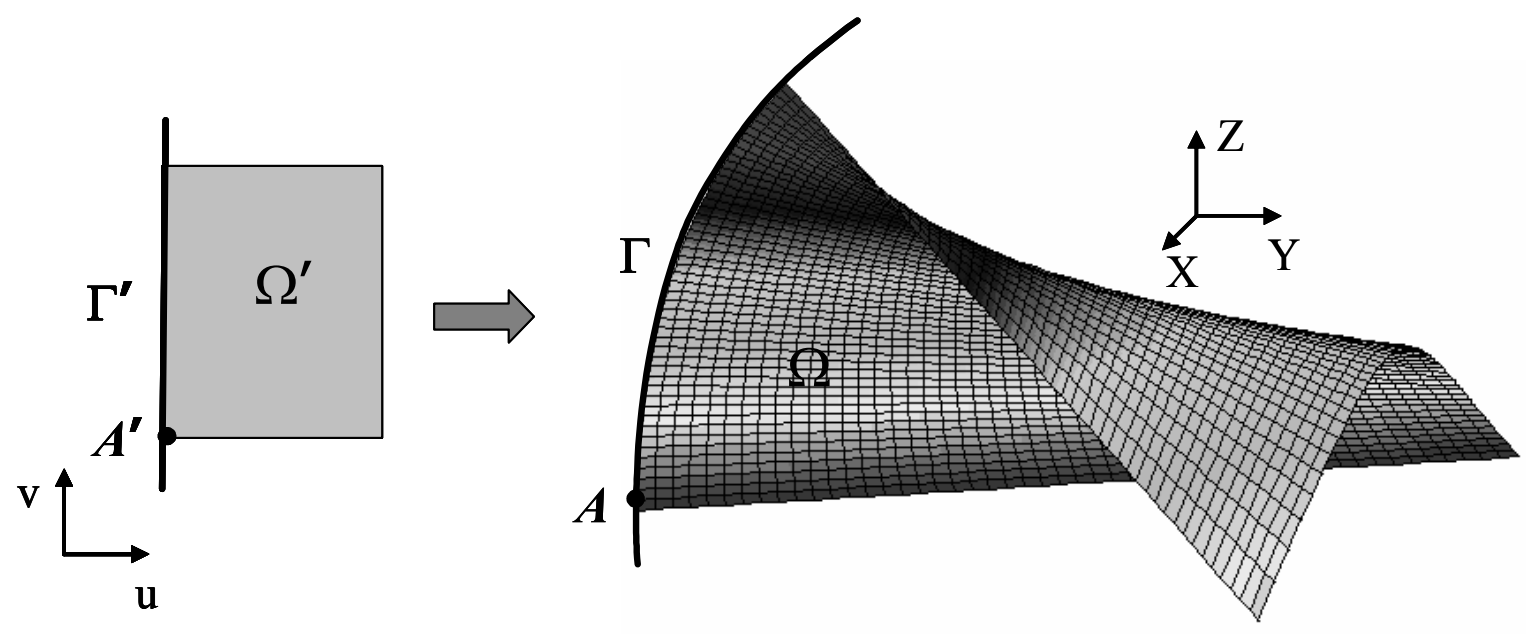

Figure 3: Generation of an applicable surface with a 3D curve. In this example a straight line $\Gamma^{\prime}$ in the $u v$-plane is mapped on a given $3 \mathrm{D}$ curve $\Gamma$.

To solve the system, we can reduce it to the following canonical form by differentiating equations (46) and (49) and using the other equations of the system to obtain

$$
\begin{aligned}
& \dot{\mathbf{R}}^{(u)}=\frac{h^{(u)} \mathbf{F}}{\dot{U} h^{(u)}+\dot{V} h^{(v)}}, \\
& \dot{\mathbf{R}}^{(v)}=\frac{h^{(v)} \mathbf{F}}{\dot{U} h^{(u)}+\dot{V} h^{(v)}}, \\
& h^{(u)}=\frac{g_{u}}{V g_{v}+U g_{u}}, \quad h^{(v)}=\frac{g_{v}}{V g_{v}+U g_{u}}, \\
& \mathbf{F}=\ddot{\mathbf{R}}-\ddot{U} \mathbf{R}^{(u)}-\ddot{V} \mathbf{R}^{(v)}, \quad g_{u}=\dddot{U}-\dddot{\mathbf{R}} \cdot \mathbf{R}^{(u)}, g_{v}=\dddot{V}-\dddot{\mathbf{R}} \cdot \mathbf{R}^{(v)},
\end{aligned}
$$

where the number of dots shows the order of derivatives with respect to $t$.

\subsection{Numerical integration}

In fact, this is a sixth-order system of ordinary differential equations with unknowns $\mathbf{R}^{(u)}$ and $\mathbf{R}^{(v)}$ with right-hand sides that depend on $\mathbf{R}^{(u)}, \mathbf{R}^{(v)}$, and given functions $U(t), V(t)$, and $\mathbf{R}(t)$ which specify the boundary curves in parametric form. In general, a solution can be obtained numerically using standard ODE solvers, e.g. widely available ones based on the Runge-Kutta method [18]. All unknowns therefore can be obtained with specified accuracy on an arbitrary grid $t_{1}, \ldots, t_{N}$ (for $\boldsymbol{\rho}(t)$ we use the last equation in (50)). With this solution, we can generate the structure of the patch on the applicable surface for $t \in\left[t_{\min }, t_{\max }\right]$.

We note that to start the numerical integration of equations (51) we need to specify initial conditions $\mathbf{R}_{0}^{(u)}=\mathbf{R}^{(u)}(0)$ and $\mathbf{R}_{0}^{(v)}=\mathbf{R}^{(v)}(0)$. They are subject to additional constraints that follow from the original system (50), (46), and (49). Indeed, the vectors $\mathbf{R}_{0}^{(u)}$ and $\mathbf{R}_{0}^{(v)}$ are dependent, 
since they satisfy the first three equations (50), which describe two orthonormal vectors. Assuming that $\left(\mathbf{r}_{u}, \mathbf{r}_{v}, \mathbf{r}_{u} \times \mathbf{r}_{v}\right)$ is a right-handed basis, we can always rotate the reference frame of the world coordinates so that in the rotated coordinates we have $\mathbf{R}_{0}^{(u)}=(1,0,0), \mathbf{R}_{0}^{(v)}=(0,1,0)$. Consistent initial conditions $\mathbf{R}_{0}^{(u)}$ and $\mathbf{R}_{0}^{(u)}$ for Eq. (51) can be obtained by application of a rotation matrix $Q(\alpha, \beta, \gamma)$ with Euler angles $\alpha, \beta$ and $\gamma$, to the vectors $(1,0,0)$ and $(0,1,0)$, respectively. We note that for some particular cases it may happen that both the functions $g_{v}$ and $g_{u}$ in Eq. (51) may be zero. In this case the equations for $h^{(u)}$ and $h^{(v)}$ can be replaced by the limiting expressions for $g_{v} \rightarrow 0, g_{u} \rightarrow 0$.

We implemented and tested an ODE solver for the above system. A simple validation was performed by comparison of the numerical solution and the following analytical solution corresponding to a cylindrical surface:

$$
X=u-u_{\min }, Y=N \cos \varphi(v), Z=N \sin \varphi(v), \varphi(v)=v / N .
$$

To reproduce this surface we started our algorithm for warping with a 3D curve with the condition that in the $(u, v)$-plane the curve $\Gamma^{\prime}$ is a straight line, $u=u_{\min }$, and the fact that the corresponding $3 \mathrm{D}$ curve for transform (52) is

$$
X(t)=0, \quad Y(t)=N \cos \varphi(t), \quad Z(t)=N \sin \varphi(t) .
$$

For this surface, we have the initial conditions for integration as $\mathbf{R}^{(u)}(0)=(-1,0,0), \mathbf{R}^{(v)}(0)=$ $\left(0,-\sin \varphi_{0}, \cos \varphi_{0}\right)$ with $\varphi_{0}=v_{\min } / N$. We integrated the forward problem (51) with these conditions using an ODE solver from MATLAB, which was based on the $4^{\text {th }}$ order Runge-Kutta method. The results were identical to the analytical solution within the tolerance specified to the solver. The numerical solutions for more complex transforms were also validated against an analytical solution obtained for a special case described in the section below.

\subsection{Special case: patch with a straight line boundary}

There is a special case, where to generate an applicable surface one can use an analytical solution instead of numerical integration. This case also serves to provide a practical demonstration of our developed theory. For this particular case we make all the assumptions we made above, plus we state that $\Gamma^{\prime}$ is a straight line, which conveniently can be parametrized in the form (44) with the following functions $U(t)$ and $V(t)$ :

$$
U(t)=U_{0}, \quad V(t)=t+V_{0}, \quad U_{0}, V_{0}=\text { const. }
$$

This case is practically important, since it corresponds to bending of a page with a straight edge to fit an arbitrary spatial curve $\Gamma: \mathbf{r}=\mathbf{R}(t)$.

Let us show that in this case

$$
\mathbf{R}^{(u)}=\frac{\ddot{\mathbf{R}} \times \dot{\mathbf{R}}}{|\ddot{\mathbf{R}}|}, \quad \mathbf{R}^{(v)}=\dot{\mathbf{R}},
$$

where $t$ is the natural parameter of curve $\Gamma$ (see (49)) is a solution of the forward problem (50). 
Consider the last equation in (50). Differentiating it with respect to $t$ we obtain:

$$
\begin{aligned}
\dot{\mathbf{R}} & =\left(U \dot{\mathbf{R}}^{(u)}+V \dot{\mathbf{R}}^{(v)}+\dot{\boldsymbol{\rho}}\right)+\dot{U} \mathbf{R}^{(u)}+\dot{V} \mathbf{R}^{(v)} \\
& =\left(U h^{(u)}+V h^{(v)}-1\right) \mathbf{w}+\mathbf{R}^{(v)}=\mathbf{R}^{(v)} .
\end{aligned}
$$

where we use the expressions for $\dot{\mathbf{R}}^{(u)}, \dot{\mathbf{R}}^{(v)}$, and $\dot{\boldsymbol{\rho}}$ via w (see 43)), the fourth equation (50), and the fact that $\dot{U}=0, \dot{V}=1$, which follows from (54). This proves the second equation in (55). To prove the first equation, we note that in the special case (54) we have $\ddot{U}=\ddot{V}=0$, and, therefore, the fifth equation (51) yields $\mathbf{F}=\ddot{\mathbf{R}}$. On the other hand, the two first equations (51) show that $\dot{\mathbf{R}}^{(u)}$ and $\dot{\mathbf{R}}^{(v)}$ are collinear with $\mathbf{F}$. Now we note that $\dot{\mathbf{R}}^{(u)}$ and $\dot{\mathbf{R}}^{(v)}$ are collinear to the normal to the surface $\mathbf{n}$ (see (10) and (36)), which is perpendicular both to $\mathbf{R}^{(u)}$ and $\mathbf{R}^{(v)}$ (see (3)). Therefore vector $\mathbf{R}^{(u)}$ is perpendicular to $\mathbf{F}=\ddot{\mathbf{R}}$. Since it is also perpendicular to $\mathbf{R}^{(v)}=\dot{\mathbf{R}}$ it should be collinear to $\ddot{\mathbf{R}} \times \dot{\mathbf{R}}$. Now we note that the norm of vector $\mathbf{R}^{(u)}$ is unity (see 43$)$ ) and the basis $\left(\mathbf{e}_{x}, \mathbf{e}_{y}, \mathbf{e}_{z}\right)$ defined as

$$
\mathbf{e}_{z}=\frac{\ddot{\mathbf{R}}}{|\ddot{\mathbf{R}}|}, \quad \mathbf{e}_{x}=\dot{\mathbf{R}}=\mathbf{R}^{(v)}, \quad \mathbf{e}_{y}=\mathbf{e}_{z} \times \mathbf{e}_{x}=\mathbf{R}^{(u)},
$$

has a right-handed orientation (we assume that our transform is just a bending without a change of orientation). This proves the first equation (55).

Figure 4 shows an example of image warping with a 3D curve using the analytical solution. We also performed warping using a more general ODE solver with proper initial conditions as described in the previous section and obtained the same results. A number of tests were performed with curves given parametrically by

$$
\widetilde{\mathbf{R}}(\theta)=(P(\theta), N \cos \theta, N \sin \theta), \quad P(\theta)=a_{1} \theta+a_{2} \theta^{2}+a_{3} \theta^{3}+a_{4} \theta^{4} .
$$

Some other than polynomial dependencies $P(\theta)$ were tested as well. To convert this parametric representation to a natural parametrization we solved equation (48) numerically with a standard ODE solver in MATLAB. The curve shown in Fig. 4 corresponds to $N=200, a_{1}=20, a_{2}=$ $10, a_{3}=10, a_{4}=-10$. In this example, the characteristics for this surface are not parallel, which is clearly seen from the graph in the upper right corner of Fig. 4. The image of the portrait of Ginevra dé Bencia by Leonardo da Vinci was fit into a rectangle in the $u v$-plane and warped with the generated surface $\mathbf{r}(u, v)$. Further, its orthographic projection was produced using pixel-bypixel mapping of the obtained transform from the $(u, v)$ to the $(x, y, z)$. These pictures are also shown in Fig. 4.

\section{Inverse problem: 3D structure recovery of applicable surfaces}

\subsection{Statement}

Here, we seek to estimate the 3D structure of an applicable surface from a single view (with known camera model) and knowledge of the undeformed $u v$-plane boundary. 


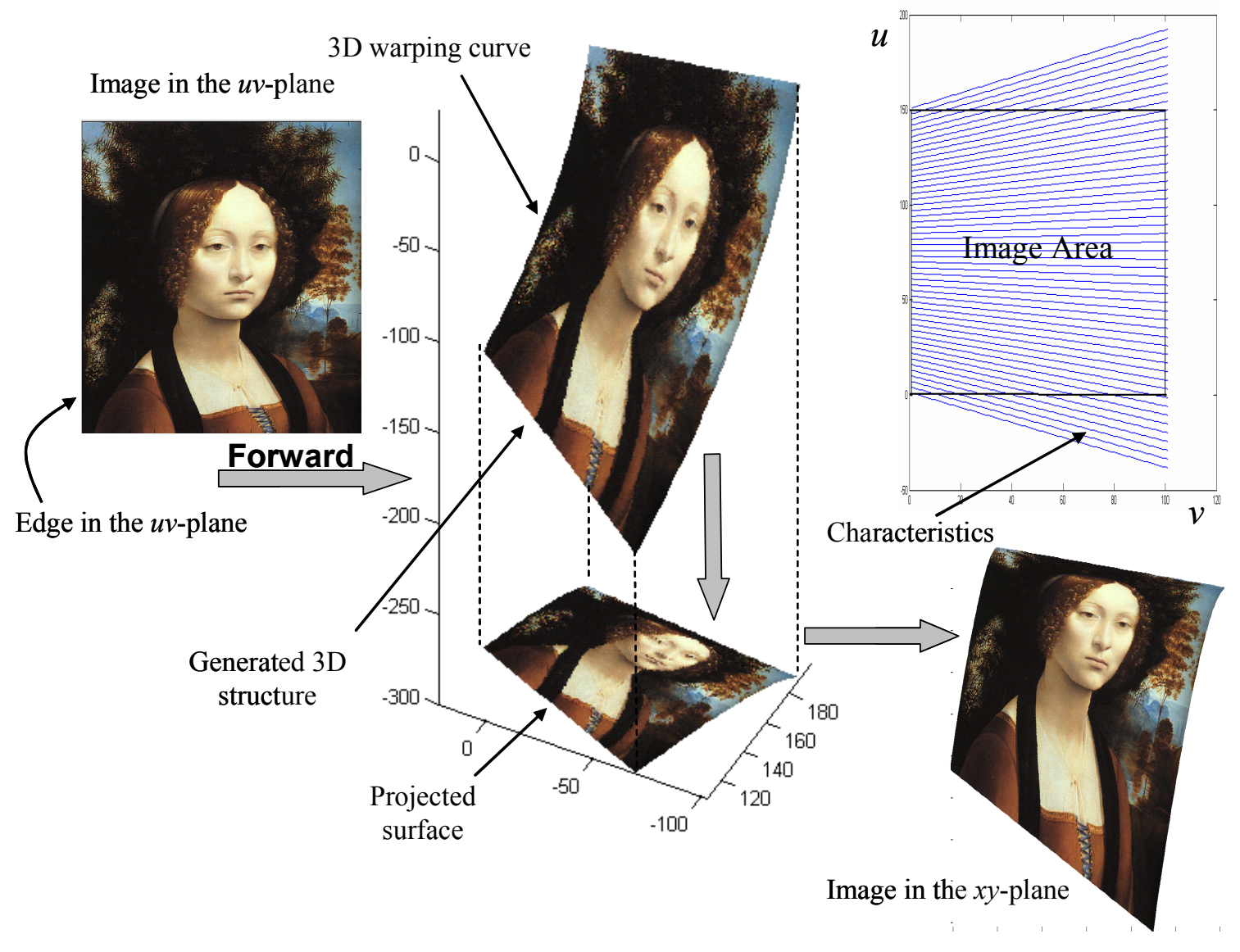

Figure 4: Illustration of the solution of the forward problem (image warping with a given 3D curve). The 3D structure is generated and then projected, which transforms the initial image in the left upper corner to the image shown in the right bottom corner. The graph in the right upper corner shows the characteristics corresponding to this case.

For any point $(x, y)$ in the image plane, we can estimate the corresponding point in the $u v$-plane and vice versa by solving the ODEs for the problem. The input parameters are the known camera model, the patch contours in the $u v$-plane and in the $x y$-, or image, plane.

We limit our consideration to patches on applicable surfaces that do not have any singularities. This means that we consider domains in the $u v$-plane, which are covered by non-intersecting characteristics. Consider, first some patch $\Omega^{\prime}$ with boundary $\partial \Omega^{\prime}$ that is a closed curve that does not coincide with the characteristic lines except at two limiting points (see Fig. 6, a)). This boundary can be subdivided into two pieces $\Gamma_{1}^{\prime}$ and $\Gamma_{2}^{\prime}$, which can be specified in parametric form as $u=U_{1}\left(s_{1}\right), v=V_{1}\left(s_{1}\right)$ for $\Gamma_{1}^{\prime}$, and $u=U_{2}\left(s_{2}\right), v=V_{2}\left(s_{2}\right)$ for $\Gamma_{2}^{\prime}$, with $u^{2}+v^{2} \neq 0$. Assume that the family of characteristics covering $\Omega^{\prime}$ corresponds to the change of parameter $t$ from $t_{\min }$ to $t_{\max }$, with $t_{\min }$ corresponding to point $A^{\prime}$ and $t_{\max }$ corresponding to point $B^{\prime}$. Hence for any $t_{*}$, such that $t_{\min }<t_{*}<t_{\max }$ there exists one point of intersection of this characteristic line with $\Gamma_{1}^{\prime}$ 


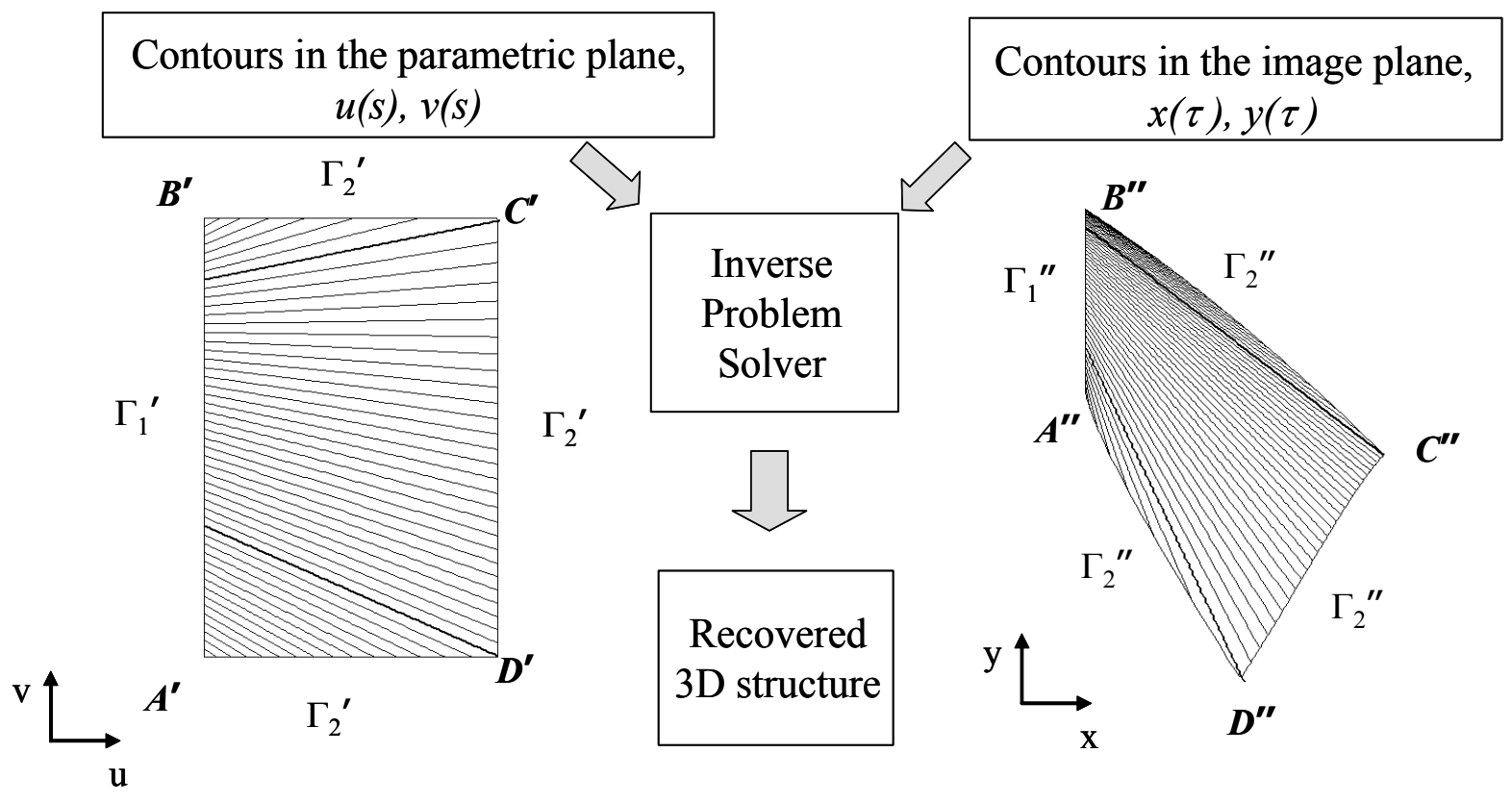

Figure 5: Schematic of the inverse problem. The input for the inverse problem solver are the equations for the curves in the parametric and image plane and some correspondence points, such as the image corners. The straight lines in the left and right graphs show the characteristics (that are obtained during the inverse problem solution).

corresponding to some value $s_{1 *}$ of the parameter of this curve $s_{1}$ and one point of intersection with $\Gamma_{2}^{\prime}$ corresponding to some value $s_{2 *}$ of the parameter of this curve $s_{2}$. This introduces two functions $s_{1}(t)$ and $s_{2}(t)$. If these functions are known, then we can find from the equation for characteristics (33) the coefficients $h^{(u)}(t)$ and $h^{(v)}(t)$ :

$$
h^{(u)}(t)=\frac{V_{2}-V_{1}}{U_{1} V_{2}-U_{2} V_{1}}, \quad h^{(u)}(t)=\frac{U_{1}-U_{2}}{U_{1} V_{2}-U_{2} V_{1}},
$$

since we assume that the functions $U_{i}\left(s_{i}\right)$ and $V_{i}\left(s_{i}\right), i=1,2$ are given explicitly. Therefore, knowledge of $s_{1}(t)$ and $s_{2}(t)$ is sufficient to build the mapping function $t(u, v)$ specified by (33) implicitly. Now we notice that the same reasoning and equations can be applied to the patches $\Omega^{\prime}$, whose boundary $\partial \Omega^{\prime}$ can partly coincide with the characteristic line (see cases b)-d) on Fig. 6). As in case a), the entire domain is covered by characteristics and functions $s_{1}(t)$ and $s_{2}(t)$ can be introduced. We will refer to such domains as "elementary". The patches that can be considered can have more complex shape than the elementary domains (e.g. see Fig. 6 e)). Any domain (elementary or non-elementary) can be subdivided into several elementary domains; in this paper we limit our consideration to the problem of recovery of elementary domains.

It is also noteworthy that the curves $\Gamma_{1}^{\prime}$ and $\Gamma_{2}^{\prime}$ need not be smooth. We assume, however, that they are piecewise continuous curves in the $u v$-plane. For example, in Fig. 5 illustrating the problem for a rectangular patch (page), the curve $\Gamma_{1}^{\prime}$ is a straight line passing through points $A^{\prime}$ and $D^{\prime}$ and 


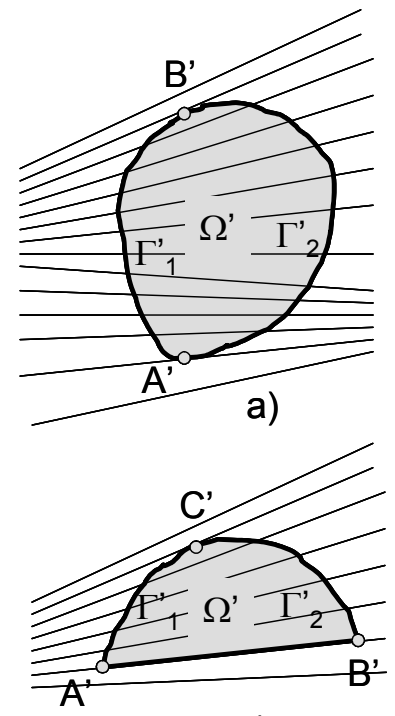

c)

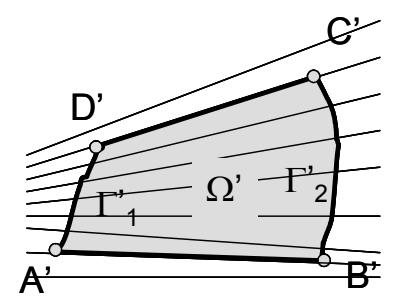

b)

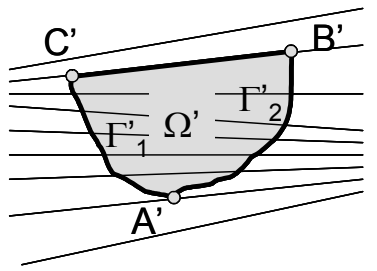

d)

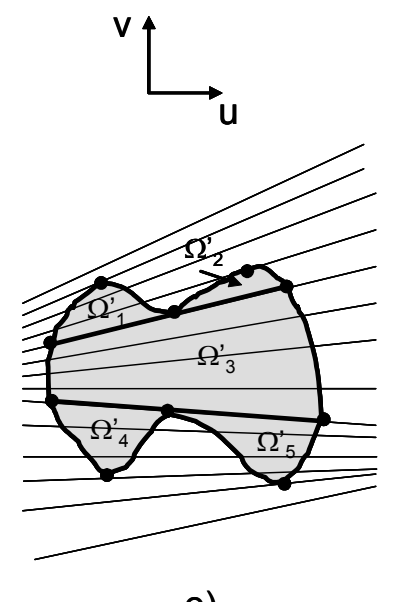

e)

Figure 6: Illustration of different types of the patch boundaries and their intersections with the characteristics.

$\Gamma_{2}^{\prime}$ consists of three smooth pieces $A^{\prime} B^{\prime}, B^{\prime} C^{\prime}$, and $C^{\prime} D^{\prime}$. When the original plane is warped to form an applicable surface the boundary curves transform to $3 \mathrm{D}$ curves $\Gamma_{1}$ and $\Gamma_{2}$, which can be described parametrically as $\mathbf{r}=\mathbf{R}_{1}(t)$ and $\mathbf{r}=\mathbf{R}_{2}(t)$, respectively.

Assume that the camera is calibrated, and the relation between the world coordinates $\mathbf{r}=(X, Y, Z)$ and coordinates of the image plane $(x, y)$ are known as $x=F^{(x)}(\mathbf{r})$ and $y=F^{(y)}(\mathbf{r})$ (via the camera model). What is also known are the curves $\Gamma_{1}^{\prime \prime}$ and $\Gamma_{2}^{\prime \prime}$ that are images of the boundary curves $\Gamma_{1}$ and $\Gamma_{2}$, i.e., we can represent them as equations. These equations are assumed to be given in the form $x=x_{1}\left(\tau_{1}\right), y=y_{1}\left(\tau_{1}\right)$ for $\Gamma_{1}^{\prime \prime}$; and $x=x_{2}\left(\tau_{2}\right), y=y_{2}\left(\tau_{2}\right)$ for $\Gamma_{2}^{\prime \prime}$. Correspondence between the boundary curves and their images is provided by a specification of the functions $\tau_{1}(t)$ and $\tau_{2}(t)$.

Thus, mathematically, the problem of recovery of the 3D structure of applicable surfaces from a single view can be formulated as a problem of determination of the unknown functions $s_{1}(t), s_{2}(t)$, $\tau_{1}(t), \tau_{2}(t), \mathbf{R}_{1}(t), \mathbf{R}_{2}(t)$, and, finally, the functions $h^{(u)}(t), h^{(v)}(t), \mathbf{R}^{(u)}(t), \mathbf{R}^{(v)}(t)$, which then can be used for mapping any point in the $u v$-plane to the $x y$-plane and back. It is also clear that if just the boundaries of the patches are given and the characteristics are not known a priori, the limiting points on the boundary (such as $A^{\prime}$ and $B^{\prime}$ in Fig. 6, a)) which break the boundary into curves $\Gamma_{1}^{\prime}$ and $\Gamma_{2}^{\prime}$ need to be first determined (this problem becomes more complex for nonelementary patches). Nevertheless, in some important cases, this problem can be simply resolved separately prior to the problem of recovery of the unknown functions, using auxiliary information. For example, if we have a rectangular page with 4 corners, then first, we usually are able to identify these corners in the image, and what is more important, in most cases the limiting points are the corner points. Of course, there is a problem; we must decide which two of the 4 corner points are 
to be taken as the limiting points. However, this problem can be resolved, by simply performing computations of all possible situations (which can be enumerated as a few cases) and comparison of the results with the actual image. Another possibility is to make this choice by using some additional information, which will become clear from the solution below. In fact, it turns out, we need only a small amount of such information.

\subsection{Equations}

The set of algebraic and differential equations describing the surface (50) can be reduced then to

$$
\begin{aligned}
& \mathbf{R}^{(u)} \cdot \mathbf{R}^{(u)}=1, \quad \mathbf{R}^{(u)} \cdot \mathbf{R}^{(v)}=0, \quad \mathbf{R}^{(v)} \cdot \mathbf{R}^{(v)}=1, \\
& \mathbf{R}_{2}=\left(U_{2}-U_{1}\right) \mathbf{R}^{(u)}+\left(V_{2}-V_{1}\right) \mathbf{R}^{(v)}+\mathbf{R}_{1}, \quad \dot{\mathbf{R}}_{i}=\dot{s}_{i}\left(U_{i}^{\prime} \mathbf{R}^{(u)}+V_{i}^{\prime} \mathbf{R}^{(v)}\right), \\
& F^{(x)}\left(\mathbf{R}_{i}\right)=x_{i}\left(\tau_{i}\right), \quad F^{(y)}\left(\mathbf{R}_{i}\right)=y_{i}\left(\tau_{i}\right), \quad i=1,2 .
\end{aligned}
$$

where the prime denotes derivatives of functions with respect to their arguments. For derivatives of functions with respect to $t$ we preserve the notation used before (dots). We have 16 equations relating the 15 unknowns $\left(\mathbf{R}^{(u)}, \mathbf{R}^{(v)}, \mathbf{R}_{1}, \mathbf{R}_{2}, s_{1}, s_{2}, \tau_{1}, \tau_{2}\right)$. As in the previous case, one equation depends on the other 15 and so the system is consistent. After $s(t), \mathbf{r}_{1}(t), \mathbf{r}_{u}(t)$, and $\mathbf{r}_{v}(t)$ are found, $h^{(u)}, h^{(v)}$, and $\rho$ can be determined from equation (59) and

$$
\boldsymbol{\rho}=\mathbf{R}_{1}-U_{1} \mathbf{R}^{(u)}-V_{1} \mathbf{R}^{(v)} .
$$

This enables determination of $t(u, v)$ and $\mathbf{r}(u, v)$, from the first and the fifth equations (43).

While they can be solved, equations (60) are not in a form convenient for numerical integration, since they are not resolved with respect to the derivatives of the unknowns and include implicit algebraic equations, which will need to be solved at each step of integration. To use standard ODE solvers we must transform the system to canonical form.

To derive this form we note that the vector $\mathbf{w}(30)$ is collinear to the normal to the surface, and we can introduce a new scalar function $k(t)$ defined as

$$
\mathbf{w}=k \mathbf{N} \text {. }
$$

The function $k$ is a function of $t$ only, since $\mathbf{w}=\mathbf{w}(t)$ and $\mathbf{N}=\mathbf{N}(t)$ (see Corollary 5, Eq. (29)). From equations (43) we have:

$$
\dot{\mathbf{R}}^{(u)}=k h^{(u)} \mathbf{N}, \quad \dot{\mathbf{R}}^{(v)}=k h^{(v)} \mathbf{N}, \quad \dot{\boldsymbol{\rho}}=-k \mathbf{N} .
$$

Consider now the equations for the projection of a 3D curve on the image plane (the last two equations in (60)). Differentiating the equations for the first curve $(i=1)$ with respect to $t$, we obtain

$$
x_{1}^{\prime} \dot{\tau}_{1}=\dot{\mathbf{R}}_{1} \cdot \nabla F^{(x)}\left(\mathbf{R}_{1}\right), \quad y_{1}^{\prime} \dot{\tau}_{1}=\dot{\mathbf{R}}_{1} \cdot \nabla F^{(y)}\left(\mathbf{R}_{1}\right) .
$$

Multiplying these equations by $x_{1}^{\prime}$ and $y_{1}^{\prime}$ and taking the sum and the difference of the results, we have

$$
\dot{\tau}_{1}=\dot{\mathbf{R}}_{1} \cdot \mathbf{a}_{1}, \quad \dot{\mathbf{R}}_{1} \cdot \mathbf{b}_{1}=0
$$


where

$$
\mathbf{a}_{1}=\frac{x_{1}^{\prime} \nabla F^{(x)}\left(\mathbf{R}_{1}\right)+y_{1}^{\prime} \nabla F^{(y)}\left(\mathbf{R}_{1}\right)}{x_{1}^{\prime 2}+y_{1}^{\prime 2}}, \quad \mathbf{b}_{1}=y_{1}^{\prime} \nabla F^{(x)}\left(\mathbf{R}_{1}\right)-x_{1}^{\prime} \nabla F^{(y)}\left(\mathbf{R}_{1}\right) .
$$

From the fifth equation (60) we have $\dot{\mathbf{R}}_{1}=\dot{s}_{1}\left(U_{1}^{\prime} \mathbf{R}^{(u)}+V_{1}^{\prime} \mathbf{R}^{(v)}\right)$, and so we can rewrite (64) as

$$
\dot{\tau}_{1}=\dot{s}_{1} \mathbf{c}_{1} \cdot \mathbf{a}_{1}, \quad \mathbf{c}_{1} \cdot \mathbf{b}_{1}=0,
$$

where

$$
\mathbf{c}_{1}=U_{1}^{\prime} \mathbf{R}^{(u)}+V_{1}^{\prime} \mathbf{R}^{(v)}
$$

Differentiation of the last equation (66) yields

$$
\dot{\mathbf{c}}_{1} \cdot \mathbf{b}_{1}+\mathbf{c}_{1} \cdot \dot{\mathbf{b}}_{1}=0 \text {. }
$$

Using (62) we express $\dot{\mathbf{c}}_{1}$ as follows:

$$
\dot{\mathbf{c}}_{1}=\dot{s}_{1}\left(U_{1}^{\prime \prime} \mathbf{R}^{(u)}+V_{1}^{\prime \prime} \mathbf{R}^{(v)}\right)+U_{1}^{\prime} \dot{\mathbf{R}}^{(u)}+V_{1}^{\prime} \dot{\mathbf{R}}^{(v)}=\dot{s}_{1} \mathbf{e}_{1}+k \mathbf{f}_{1},
$$

where

$$
\mathbf{e}_{1}=U_{1}^{\prime \prime} \mathbf{R}^{(u)}+V_{1}^{\prime \prime} \mathbf{R}^{(v)}, \quad \mathbf{f}_{1}=U_{1}^{\prime} h^{(u)}+V_{1}^{\prime} h^{(v)} .
$$

To obtain an expression for $\dot{\mathbf{b}}_{1}$ we will use the following notation for the second rank Hessian of the scalar function $F(\mathbf{r})$ :

$$
\nabla \nabla F(\mathbf{r})=\left(\begin{array}{ccc}
\frac{\partial^{2}}{\partial x^{2}} F(\mathbf{r}) & \frac{\partial^{2}}{\partial x \partial y} F(\mathbf{r}) & \frac{\partial^{2}}{\partial x \partial z} F(\mathbf{r}) \\
\frac{\partial^{2}}{\partial x \partial y} F(\mathbf{r}) & \frac{\partial^{2}}{\partial y^{2}} F(\mathbf{r}) & \frac{\partial^{2}}{\partial y \partial z} F(\mathbf{r}) \\
\frac{\partial^{2}}{\partial x \partial z} F(\mathbf{r}) & \frac{\partial^{2}}{\partial y \partial z} F(\mathbf{r}) & \frac{\partial^{2}}{\partial z^{2}} F(\mathbf{r})
\end{array}\right) .
$$

The dot product of this tensor with some vector is a vector. If the vector components are arranged as a column-vector, then the resulting vector is a product of the Hessian matrix by the input vector. So we have:

$$
\dot{\mathbf{b}}_{1}=\dot{\tau}_{1} \mathbf{d}_{1}+\mathbf{G}_{1} \cdot \dot{\mathbf{R}}_{1}=\dot{s}_{1}\left[\left(\mathbf{c}_{1} \cdot \mathbf{a}_{1}\right) \mathbf{d}_{1}+\mathbf{G}_{1} \cdot \mathbf{c}_{1}\right],
$$

where

$$
\mathbf{d}_{1}=y_{1}^{\prime \prime} \nabla F^{(x)}\left(\mathbf{R}_{1}\right)-x_{1}^{\prime \prime} \nabla F^{(y)}\left(\mathbf{R}_{1}\right), \quad \mathbf{G}_{1}=y_{1}^{\prime} \nabla \nabla F^{(x)}\left(\mathbf{R}_{1}\right)-x_{1}^{\prime} \nabla \nabla F^{(y)}\left(\mathbf{R}_{1}\right) .
$$

Substituting the expressions (69) and (72) into equation (68) we obtain the following relation between $\dot{s}_{1}$ and $k$ :

$$
\dot{s}_{1}=-\frac{k \mathbf{f}_{1} \cdot \mathbf{b}_{1}}{\mathbf{e}_{1} \cdot \mathbf{b}_{1}+\mathbf{c}_{1} \cdot\left[\left(\mathbf{c}_{1} \cdot \mathbf{a}_{1}\right) \mathbf{d}_{1}+\mathbf{G}_{1} \cdot \mathbf{c}_{1}\right]} .
$$


A similar relation holds for $\dot{s}_{2}$, since we can replace subscript 1 with subscript 2 in equations (63)(74).

Now note that equations (60) are invariant with respect to an arbitrary one-to-one transform $t \rightarrow f(t)$. In other words, the function $t$ can be defined arbitrarily, as long as the value of this function is specific for each characteristic. For example, it can be defined as one of the curve parameters $s_{1}, s_{2}, \tau_{1}$, or $\tau_{2}$, as long as they are one-to-one mappings. It may be more convenient to introduce some scaling factor, and, so, without any loss of generality we can define $t$ as a linear function of, say, $s_{1}$. This definition is equivalent to the statement that the rate of change of $s_{1}$ is a constant, sio, or

$$
s_{1}=\dot{s}_{10}\left(t-t_{\min }\right)+s_{10}
$$

where $s_{10}$ is the value of $s_{1}$ corresponding $t=t_{\min }$. Using this definition of $t$ and equations (66) and (74) written for each boundary curve, we can write down the following ODEs:

$$
\dot{s}_{1}=h_{1}, \quad \dot{s}_{2}=h_{2}, \quad \dot{\tau}_{1}=g_{1}, \quad \dot{\tau}_{2}=g_{2},
$$

where

$$
\begin{aligned}
& h_{1}=\dot{s}_{10}=\text { const, } \\
& h_{2}=-\frac{k \mathbf{f}_{2} \cdot \mathbf{b}_{2}}{\mathbf{e}_{2} \cdot \mathbf{b}_{2}+\mathbf{c}_{2} \cdot\left[\left(\mathbf{c}_{2} \cdot \mathbf{a}_{2}\right) \mathbf{d}_{2}+\mathbf{G}_{2} \cdot \mathbf{c}_{2}\right]}, \\
& g_{i}=h_{i} \mathbf{c}_{i} \cdot \mathbf{a}_{i}, \quad i=1,2, \\
& k=-\frac{\mathbf{e}_{1} \cdot \mathbf{b}_{1}+\mathbf{c}_{1} \cdot\left[\left(\mathbf{c}_{1} \cdot \mathbf{a}_{1}\right) \mathbf{d}_{1}+\mathbf{G}_{1} \cdot \mathbf{c}_{1}\right]}{\mathbf{f}_{1} \cdot \mathbf{b}_{1}} h_{1}, \\
& h^{(u)}=\frac{V_{2}-V_{1}}{U_{1} V_{2}-U_{2} V_{1}}, h^{(v)}=\frac{U_{1}-U_{2}}{U_{1} V_{2}-U_{2} V_{1}}, \quad \mathbf{N}=\mathbf{R}^{(u)} \times \mathbf{R}^{(v)}, \\
& \mathbf{R}_{i}=U_{i} \mathbf{R}^{(u)}+V_{i} \mathbf{R}^{(v)}+\boldsymbol{\rho}, \quad i=1,2, \\
& \mathbf{a}_{i}=\frac{x_{i}^{\prime} \nabla F^{(x)}\left(\mathbf{R}_{i}\right)+y_{i}^{\prime} \nabla F^{(y)}\left(\mathbf{R}_{i}\right)}{x_{i}^{\prime 2}+y_{i}^{\prime 2}}, \quad \mathbf{b}_{i}=y_{i}^{\prime} \nabla F^{(x)}\left(\mathbf{R}_{i}\right)-x_{i}^{\prime} \nabla F^{(y)}\left(\mathbf{R}_{i}\right), \quad i=1,2, \\
& \mathbf{c}_{i}=U_{i}^{\prime} \mathbf{R}^{(u)}+V_{i}^{\prime} \mathbf{R}^{(v)}, \mathbf{d}_{i}=y_{i}^{\prime \prime} \nabla F^{(x)}\left(\mathbf{R}_{i}\right)-x_{i}^{\prime \prime} \nabla F^{(y)}\left(\mathbf{R}_{i}\right), \quad i=1,2, \\
& \mathbf{e}_{i}=U_{i}^{\prime \prime} \mathbf{R}^{(u)}+V_{i}^{\prime \prime} \mathbf{R}^{(v)}, \mathbf{f}_{i}=\left(U_{i}^{\prime} h^{(u)}+V_{i}^{\prime} h^{(v)}\right) \mathbf{N}^{\prime}, i=1,2, \\
& \mathbf{G}_{i}=y_{i}^{\prime} \nabla \nabla F^{(x)}\left(\mathbf{R}_{i}\right)-x_{i}^{\prime} \nabla \nabla F^{(y)}\left(\mathbf{R}_{i}\right), \quad i=1,2 .
\end{aligned}
$$

Now we note that the system (62) and (76) is closed with respect to variables $\left(s_{1}, s_{2}, \tau_{1}, \tau_{2}\right.$, $\left.\mathbf{R}^{(u)}, \mathbf{R}^{(v)}, \boldsymbol{\rho}\right)$. Moreover it is written in a canonical form, since it is resolved with respect to derivatives, and the right hand sides of the equations are explicit functions of variables $\left(s_{1}, s_{2}, \tau_{1}\right.$, $\left.\tau_{2}, \mathbf{R}^{(u)}, \mathbf{R}^{(v)}, \boldsymbol{\rho}\right)$. Thus, it can be solved numerically using standard methods, if proper initial conditions are imposed. In some particular cases the system can be substantially simplified (e.g. for a rectangular patch the boundaries in the $u v$-plane are straight lines, $\mathbf{e}_{i}=\mathbf{0}, i=1,2$, or for orthographic projection $F^{(x)}$ and $F^{(y)}$ are linear functions of world coordinates, and so $\mathbf{G}_{i}=\mathbf{0}$, 
$i=1,2)$. However such simplifications do not prevent numerical solution and the same ODE solver should be used for general or special cases.

Another issue is related to the treatment of piecewise smooth boundaries. In this case, the derivatives of the boundary curves can be discontinuous and should be taken according to the direction of integration. So one can break the integration and perform it in pieces over the smooth parts of the boundary and use the condition that the functions $\mathbf{R}^{(u)}, \mathbf{R}^{(v)}, k, h^{(u)}$, and $h^{(v)}$ should be smooth functions of $t$ at the corners, since these functions characterize the applicable (smooth) surface. For example, for the case shown in Fig. 5 we can break the integration from $t_{\min }$ to $t_{\max }$ into three pieces: from $t_{\min }$ to $t_{1}$, from $t_{1}$ to $t_{2}$, and from $t_{2}$ to $t_{\max }$, where $t_{\min }, t_{1}, t_{2}$, and $t_{\max }$ correspond to the corners of the rectangular patch $A^{\prime \prime}, B^{\prime \prime}, C^{\prime \prime}$, and $D^{\prime \prime}$, respectively. Since functions $\left(s_{1}, s_{2}, \tau_{1}, \tau_{2}, \mathbf{R}^{(u)}, \mathbf{R}^{(v)}, \boldsymbol{\rho}\right)$ are continuous functions of $t$, the solution at the end point of the interval can be imposed as the initial condition for the next interval. Determination of the intervals of smoothness $\left(t_{\mathrm{min}}, t_{1}\right),\left(t_{1}, t_{2}\right)$, etc. can be performed automatically in the process of integration, since parametrization of the boundary curve in the $u v$-plane can be made in such a way that the corner points correspond to specific convenient values of the curve parameter (for example, we parametrized the boundary curve for a rectangular patch so that corners $A^{\prime}, B^{\prime}$, and $C^{\prime}$ correspond to $s_{2}=0,1$, and 2 , respectively, see Fig. 5, so $t_{1}$ and $t_{2}$ were the values of $t$ at which $s_{2}$ was equal to 1 and 2).

\subsection{Initial conditions}

To solve the initial value problem for the system of ODEs (62), (76) we should specify the initial values of the unknowns $\left(s_{1}, s_{2}, \tau_{1}, \tau_{2}, \mathbf{R}^{(u)}, \mathbf{R}^{(v)}, \boldsymbol{\rho}\right)$ at $t=t_{\text {min }}$. We will denote these values with subscript zero. Furthermore, we assume a natural parametrization of the boundary curves that starts from this point, and impose the conditions:

$$
s_{10}=s_{20}=\tau_{10}=\tau_{20}=0 .
$$

The remaining 9 initial conditions (vectors $\mathbf{R}_{0}^{(u)}, \mathbf{R}_{0}^{(v)}$, and $\boldsymbol{\rho}_{0}$ ) are not arbitrary, and should satisfy certain relations. First, we recall that vectors $\mathbf{R}_{0}^{(u)}, \mathbf{R}_{0}^{(v)}$, and $\mathbf{N}_{0}=\mathbf{R}_{0}^{(u)} \times \mathbf{R}_{0}^{(v)}$ form a right-handed basis. This basis can be obtained by application of the rotation matrix $Q_{0}$ to the standard unit vectors:

$$
\mathbf{R}_{0}^{(u)}=Q_{0}\left(\begin{array}{l}
1 \\
0 \\
0
\end{array}\right)=Q_{0} \mathbf{i}_{x}, \quad \mathbf{R}_{0}^{(v)}=Q_{0}\left(\begin{array}{l}
0 \\
1 \\
0
\end{array}\right)=Q_{0} \mathbf{i}_{y}, \quad \mathbf{N}_{0}=Q_{0}\left(\begin{array}{l}
0 \\
0 \\
1
\end{array}\right)=Q_{0} \mathbf{i}_{z}, .
$$

where the entries of $Q_{0}$ depend only on the three Euler rotation angles $\alpha_{0}, \beta_{0}$, and $\gamma_{0}$ :

$Q_{0}=\left(\begin{array}{ccc}\cos \alpha_{0} \cos \beta_{0} \cos \gamma_{0}-\sin \alpha_{0} \sin \gamma_{0} & \sin \alpha_{0} \cos \beta_{0} \cos \gamma_{0}+\cos \alpha_{0} \sin \gamma_{0} & \sin \beta_{0} \cos \gamma_{0} \\ \cos \alpha_{0} \cos \beta_{0} \sin \gamma_{0}+\sin \alpha_{0} \cos \gamma_{0} & \sin \alpha_{0} \cos \beta_{0} \sin \gamma_{0}-\cos \alpha_{0} \cos \gamma_{0} & \sin \beta_{0} \sin \gamma_{0} \\ -\cos \alpha_{0} \sin \beta_{0} & \sin \alpha_{0} \sin \beta_{0} & \cos \beta_{0}\end{array}\right)$.

So we can see that

$$
\mathbf{R}_{0}^{(u)}=\mathbf{R}_{0}^{(u)}\left(\alpha_{0}, \beta_{0}, \gamma_{0}\right), \quad \mathbf{R}_{0}^{(v)}=\mathbf{R}_{0}^{(v)}\left(\alpha_{0}, \beta_{0}, \gamma_{0}\right), \quad \mathbf{N}_{0}=\mathbf{N}_{0}\left(\alpha_{0}, \beta_{0}, \gamma_{0}\right),
$$


and, in fact, one need only specify the 6 values, $\alpha_{0}, \beta_{0}, \gamma_{0}$, and $\boldsymbol{\rho}_{0}$ to enable solution of the initial value problem.

The number of unknown initial values can be reduced further. Relations between the initial values depend on the problem to be solved. To illustrate the reduction let us consider the case illustrated in Figures 6, a) and 5. Here, at the starting point $A^{\prime}$ the boundary curves intersect and so we have

$$
\begin{aligned}
\mathbf{R}_{10} & =\mathbf{R}_{20}=\mathbf{r}_{0}, \quad U_{10}=U_{20}=U_{0}, \quad V_{10}=V_{20}=V_{0}, \\
x_{10} & =x_{20}=F^{(x)}\left(\mathbf{r}_{0}\right), \quad y_{10}=y_{20}=F^{(y)}\left(\mathbf{r}_{0}\right) .
\end{aligned}
$$

The last two equations specify the unknown $\mathbf{r}_{0}$ as a function of the one free parameter, which is denoted as $\xi_{0}$ :

$$
\mathbf{r}_{0}=\mathbf{r}_{0}\left(\xi_{0}\right)
$$

Note that for orthographic projection, where $F^{(x)}\left(\mathbf{r}_{0}\right)=\mathbf{i}_{x} \cdot \mathbf{r}_{0}, F^{(y)}\left(\mathbf{r}_{0}\right)=\mathbf{i}_{y} \cdot \mathbf{r}_{0}$, the free parameter can be selected as $\xi_{0}=\mathbf{i}_{z} \cdot \mathbf{r}_{0}=z_{0}$, and, since in this case the image does not depend on the selection of $z_{0}$, any solution $\mathbf{r}=\mathbf{r}(u, v)$ is equivalent to the solution $\mathbf{r}=\mathbf{r}(u, v)+\mathbf{i}_{z} z_{0}$. Therefore, without any loss of generality, one can set $\xi_{0}=z_{0}=0$, keeping in mind that the 3D structure can be determined up to an arbitrary shift of the $z$-axis.

For an arbitrary projection, equation (83) specifies $\rho_{0}$ as a function of $\xi_{0}$ and the initial Euler angles due to equation

$$
\boldsymbol{\rho}_{0}\left(\alpha_{0}, \beta_{0}, \gamma_{0}, \xi_{0}\right)=\mathbf{r}_{0}\left(\xi_{0}\right)-U_{0} \mathbf{R}_{0}^{(u)}\left(\alpha_{0}, \beta_{0}, \gamma_{0}\right)-V_{0} \mathbf{R}_{0}^{(u)}\left(\alpha_{0}, \beta_{0}, \gamma_{0}\right) .
$$

This reduces the number of parameters specifying initial conditions from 6 to 4 . A further reduction of the initial parameters occurs due to the orthogonality conditions

$$
\mathbf{c}_{10} \cdot \mathbf{b}_{10}=0, \quad \mathbf{c}_{20} \cdot \mathbf{b}_{20}=0
$$

Here, according to relations (67), the vectors $\mathbf{c}_{10}$ and $\mathbf{c}_{20}$ are functions of the initial Euler angles $\alpha_{0}, \beta_{0}$, and $\gamma_{0}$, while $\mathbf{b}_{10}$ and $\mathbf{b}_{20}$ are functions of $\mathbf{r}_{0}$ or $\xi_{0}$ (see (65) and (83)). For orthographic projection we have $\nabla F^{(x)}=\mathbf{i}_{x}, \nabla F^{(y)}=\mathbf{i}_{y}$, and in this particular case $\mathbf{b}_{10}$ and $\mathbf{b}_{20}$ do not depend on $\xi_{0}$. If the starting point is a corner point, such as shown in Fig. 5, the relations (85) are independent and reduce the number of initial parameters by 2 . For example, $\gamma_{0}$ and $\xi_{0}$ can be selected as independent initial parameters, while angles $\alpha_{0}$ and $\beta_{0}$ can be expressed as the following functions:

$$
\beta_{0}=B_{0}\left(\gamma_{0}, \xi_{0}\right), \quad \alpha_{0}=A_{0}\left(\gamma_{0}, \xi_{0}\right)
$$

If the boundary curves match at the initial point smoothly, one can replace the second equation in (85) with a condition that follows from the equality of the derivatives along the boundary curves (we will not elaborate this case here, since our purpose now is just give an idea of how the number of initial parameters can be efficiently reduced). In the Appendix we derive explicit forms for computation of $B_{0}\left(\gamma_{0}, \xi_{0}\right)$ and $A_{0}\left(\gamma_{0}, \xi_{0}\right)$. 


\subsection{Boundary conditions}

The above analysis shows that while the number of parameters specifying initial conditions can be substantially reduced to two parameters (for a general camera model) or to one parameter (in the case of orthographic projection), some additional information to start the integration process. Moreover, for any set of initial parameters we also should select the correct solutions appropriate to the situation due to the non-uniqueness discussed above. To perform this task, information about correspondence of some points on the boundary curves in the $u v$ - and $x y$-planes can be used. Such feature points can appear naturally. For example, if the domain is a patch with corners that can be easily identified in the image (e.g. see Fig. 5), then instead of the unknown initial parameters we can impose the boundary value problem for the set of ODEs (62) and (76). Assume that the feature points are located on curves $\Gamma_{2}^{\prime}$ and $\Gamma_{2}^{\prime \prime}$ (e.g. points $B^{\prime}$ and $B^{\prime \prime}$ on Fig. 5). Due to a freedom in the parametrization of these curves, we can define $s_{2}$ and $\tau_{2}$ in such a way that the feature point correspond to $s_{2}=1$ and $\tau_{2}=1$. Assume, now, that we start integration with some arbitrary initial parameters $\left(\gamma_{0}, \xi_{0}, m\right)$, where $m$ is some index of solution, $m=1,2, \ldots$ (due to the multiplicity of solutions for a given $\left.\left(\gamma_{0}, \xi_{0}\right)\right)$ and stop the integration when either $s_{2}$ or $\tau_{2}$ reaches 1 . The deficiency of the solution at the feature point then can be characterized by the following objective function

$$
J\left(\gamma_{0}, \xi_{0}, m\right)=\left[\left(1-s_{2}\right)^{2}+\left(1-\tau_{2}\right)^{2}\right]^{1 / 2} .
$$

Due to errors in integration, data interpolation, or quantization etc., we should not expect that this function will be exactly 0 for the proper set $\left(\gamma_{0}, \xi_{0}, m\right)$. However, $J\left(\gamma_{0}, \xi_{0}, m\right)$ is locally convex and has a strong minimum, so we can state the problem of determination of the initial parameters as a minimization problem for this function. The computation of $J\left(\gamma_{0}, \xi_{0}, m\right)$ for each set of arguments requires the solution of the system of ODEs; this is known as the shooting method for solution of the boundary value problem. If more information is available (e.g. we can identify all the corners), the objective function can be constructed in a way that minimizes the deficiency of the solution at all feature points.

\subsection{Ambiguities}

The method relies on the boundary information of the patch in the image plane; since some deformations can lead us to the same images of the boundary, we have ambiguities. For example, consider a cylindrical surface

$$
X(u, v)=u, \quad Y(u, v)=f(v), \quad Z(u, v)=g(v),
$$

with some functions $f$ and $g$, which make this surface applicable. Assume that in the $u v$-plane the patch is a rectangle bounded by lines $u=u_{\min }, v=v_{\min }, u=u_{\max }$, and $v=v_{\max }$. For orthographic projection the image of this patch will be a rectangle bounded by lines $x=u_{\min }$, $y=f\left(v_{\min }\right), x=u_{\max }$, and $y=f\left(v_{\max }\right)$. This shows that for any two functions $f_{1}(v)$ and $f_{2}(v)$, such that $f_{1}\left(v_{\min }\right)=f_{2}\left(v_{\min }\right)$ and $f_{1}\left(v_{\max }\right)=f_{2}\left(v_{\max }\right)$ the patch in the image plane will be bounded by the same rectangle, and it is impossible to uncover $f_{1}(v)$ based only on the boundary information. 
The problem of classification of all ambiguities and the methods to treat these cases is a subject for a separate study. To resolve the ambiguities we can use some additional useful cues such as texture or shading.

\subsection{Example problem}

Despite possible ambiguities we should state that these are very special cases. In general the inverse problem can be resolved. The reconstruction of the 3D structure and texture mapping from the $x y$ to $u v$ - plane (unwarping) can be efficiently performed using the boundary information from a single view. To test the solution we performed the following numerical example.

We took a rectangular patch in the $u v$-plane $\left(u_{\min }=v_{\min }=1, u_{\max }=151, v_{\max }=101\right)$, and generated a patch on the applicable surface using the analytical solution of the forward problem (55). The 3D curve used for forward problem was (58) with $N=200, a_{1}=20, a_{2}=10, a_{3}=$ $10, a_{4}=-10$ (see Fig. 4). Further we took the orthographic projection of the patch boundaries and discretized each side of the patch with 300 points, which were used as input $(x, y)$ data for the inverse problem solver.

The test of the inverse problem solver was performed in several stages. The goal for the first stage was to ensure that the system of ODEs (62), (76), and (77) is derived correctly, and it can be solved with proper initial conditions. Since the forward problem was simulated, we knew the information about the characteristics - which corner corresponds to $t_{\min }$ and all functions which specify the $3 \mathrm{D}$ structure $\left(\mathbf{R}_{0}(t), \mathbf{R}_{0}(t), \boldsymbol{\rho}_{0}(t), h_{u}(t)\right.$, and $\left.h_{v}(t)\right)$. So we know the initial values $\left(\mathbf{R}_{0}^{(u)}, \mathbf{R}_{0}^{(v)}, \boldsymbol{\rho}_{0}\right)$ for the start of integration. We run the ODE solver (standard MATLAB function) with the specified right-hand sides (77) and these initial conditions, and compared the obtained solution for all functions $\left(\mathbf{R}_{0}(t), \mathbf{R}_{0}(t), \boldsymbol{\rho}_{0}(t), h_{u}(t)\right.$, and $\left.h_{v}(t)\right)$ with the analytical solution for the forward problem at given values of $t$. This solution was close to the analytical solution within the specified tolerance, which was varied between $\epsilon=10^{-4}-10^{-8}$, except in some small vicinity of the end point, at which the functions specifying the right hand side (77) have a singularity due to intersection of the boundary curves. This problem, however, can be avoided by extrapolation of all functions of $t$ found for $\left[t_{\min }, t_{\max }-t_{\epsilon}\right]$ with a good accuracy to the small interval $\left[t_{\max }-t_{\epsilon}, t_{\max }\right]$ near the end point. This is possible to do since $\mathbf{R}_{0}(t), \mathbf{R}_{0}(t), \boldsymbol{\rho}_{0}(t), h_{u}(t)$, and $h_{v}(t)$ are smooth differentiable functions. We note that because the boundary in the present case was piecewise smooth, we used the procedure which stops the integration when parameters $s_{2}$ or $\tau_{2}$ reach the values corresponding to the corner, and restarted the integration with the curve parameters for the next smooth piece (with the direction for derivatives taken accordingly; the derivatives of the boundary curves were computed using numerical differentiation of the tabulated boundary curves).

After we were ensured that the ODE solver produces accurate results for correct initial values, we considered the problem of recovery of these values from information about the boundary curve. As we noticed earlier, for the orthographic projection parameter $\xi_{0}=z_{0}$ can be selected arbitrarily and we set it to zero. We note that in the test case expressions for the Euler angles $\alpha_{0}$ and $\beta_{0}$ via $\gamma_{0}$ can be found analytically, since for orthographic projection we have $\chi_{10}=\chi_{20}=\pi / 2$, and for the initial point where the boundary curves $\Gamma_{1}^{\prime}$ and $\Gamma_{2}^{\prime}$ intersect at $90^{\circ}$ (rectangular patch in the $u v$ plane) we have $\varphi_{10}=\pi / 2$ and $\varphi_{20}=0$. In this case equations (98) can be written in the following matrix-vector form: 


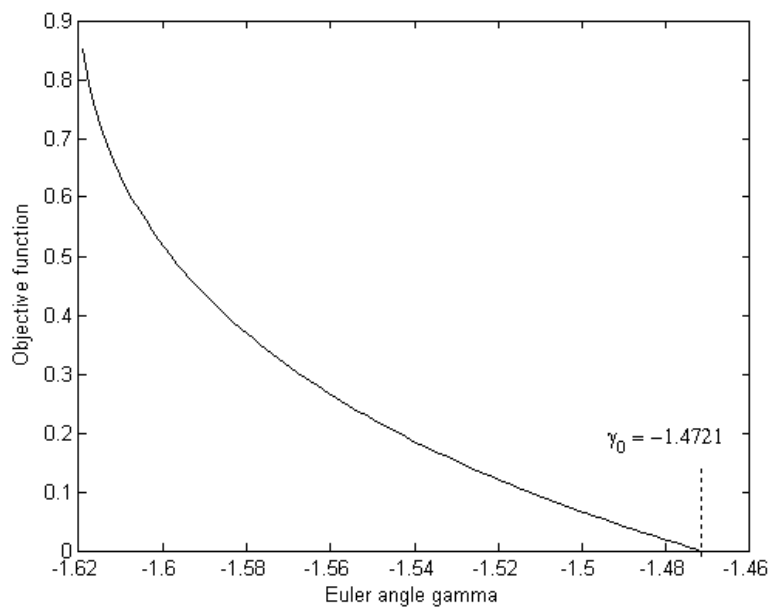

Figure 7: The objective function 87 for the example problem. The true value of the Euler angle $\gamma_{0}$ $=-1.4721$ is shown.

$$
\left(\begin{array}{cc}
-\cos \left(\psi_{10}-\gamma_{0}\right) \cos \beta_{0} & \sin \left(\psi_{10}-\gamma_{0}\right) \\
\sin \left(\psi_{20}-\gamma_{0}\right) & \cos \left(\psi_{20}-\gamma_{0}\right) \cos \beta_{0}
\end{array}\right)\left(\begin{array}{c}
\sin \alpha_{0} \\
\cos \alpha_{0}
\end{array}\right)=0
$$

Solution of these equations with respect to $\left(\sin \alpha_{0}, \cos \alpha_{0}\right)$ treated as an independent vector exists only if the determinant of the matrix is zero, which leads to equation

$$
\cos ^{2} \beta_{0}=-\tan \left(\psi_{20}-\gamma_{0}\right) \tan \left(\psi_{10}-\gamma_{0}\right)
$$

Furthermore, solution of this equation exists only if

$$
0 \leqslant g \leqslant 1, \quad g=\tan \left(\psi_{20}-\gamma_{0}\right) \tan \left(\gamma_{0}-\psi_{10}\right) .
$$

In this case we have four roots:

$$
\beta_{0}= \pm \arccos ( \pm \sqrt{g}) \text {. }
$$

where \pm can be selected in arbitrary combination. According (99) each $\beta_{0}$ then produces two values of $\alpha_{0}$

$$
\alpha_{0}=-\frac{\pi}{2}-\arctan \left[\cot \left(\psi_{10}-\gamma_{0}\right) \cos \beta_{0}\right]+\pi n, \quad n=0, \pm 1, \ldots, \quad-\pi<\alpha_{0} \leqslant \pi .
$$

So for any given $\gamma_{0}$ we have 8 possible sets of the Euler angles. By comparison with the initial Euler angles for the exact solution, we found that the correct solution is among that 8 solutions.

Finally we performed solution of the boundary value problem, where we attempted to uncover $\gamma_{0}$ and $m$ using minimization of the objective function (87). For the present example the range of 
$\gamma_{0}$ is between $\psi_{10}=-1.6208$ and $\psi_{20}-\pi=-1.4706$ where solution of equation (91) exists (due to $\pi$-periodicity $\gamma_{0}+\pi$ is also a solution), so this provides the bounds for the roots. The true value $\gamma_{0}=-1.4721$ (from the forward problem) and we can see that the zero of the objective function is close to this value (this depends on the specified tolerance for the ODE solver and the tolerance to the approximation near the corner point). Our computations were stable for $-1.6208<$ $\gamma_{0}<-1.4721$, while for $-1.4706<\gamma_{0}<-1.4721$ the objective function was not computable (due to the singularities in the right hand side for $s_{2}<1$ and $\tau_{2}<1$ ). However, our method of computation of the objective function is compatible with the standard MATLAB root finding routine, which converged to the accurate solution in a few iterations ( 15 for accuracy $10^{-4}$ ).

Note that due to several solutions corresponding to the same $\gamma_{0}$ we compared the objective functions obtained for all possible $\alpha_{0}$ and $\beta_{0}$. This comparison showed that 2 out of 8 solutions provide the minimum of the objective function for the same $\gamma_{0}$. We also checked that if $\gamma_{0}$ provides a minimum of the objective function, then the symmetrical minimum exists for $\gamma_{0}+\pi$. Here again, only 2 out of 8 solutions deliver the zero of the objective function. So, totally, we found 4 different combinations of the Euler angles $\left(\alpha_{0}, \beta_{0}, \gamma_{0}\right)$ which minimize the objective function. We can note now that transform $\left(\alpha_{0}, \beta_{0}, \gamma_{0}\right) \rightarrow\left(\alpha_{0}+\pi,-\beta_{0}, \gamma_{0}+\pi\right)$ does not change the rotation matrix $Q_{0}(80)$ and so such solutions should be considered as identical (generate the same surface). This leave us with only 2 different solutions. As mentioned above, for the orthographic projection we always have at least two different solutions, $(X(u, v), Y(u, v), Z(u, v))$ and its mirror $(X(u, v), Y(u, v),-Z(u, v))$. This also means that while the $3 \mathrm{D}$ structure uncovered is not unique (we also do not have any information about the depth), the transform from the image to the parametric plane (and back) is unique and enables solution of the unwarping problem.

Finally we note that the quality of the synthetic image warping/unwarping depends on the surface and projection. For the case described above the solution of the inverse problem (use as the input the image in the right bottom corner of Fig. 4) practically coincides with the exact solution (the image in the left top corner of Fig. 4 is the output). Figure 8 illustrates the unwarped pixelby-pixel image as the end point of the unwarping process in the $x y$-plane. We ran the algorithm for small fonts. The original image has the same font size everywhere and with the forward algorithm we warp the image. The unwarped image has uniform font size everywhere, lines are parallel and right angles are preserved. The output is noisy (especially at the top), since in the image some information was lost.

\section{Acknowledgments}

Support of NSF awards 99-87944 and 00-86075 gratefully acknowledged.

\section{Appendix}

To derive explicit forms suitable for computation of functions $B_{0}\left(\gamma_{0}, \xi_{0}\right)$ and $A_{0}\left(\gamma_{0}, \xi_{0}\right)$ (see (86)) let us introduce the following unit vectors, assuming that the starting point is the corner point, in which case these vectors are not collinear:

$$
\mathbf{j}_{i 0}=\frac{U_{i 0}^{\prime} \mathbf{i}_{x}+V_{i 0}^{\prime} \mathbf{i}_{y}}{U_{i 0}^{\prime 2}+V_{i 0}^{\prime 2}}=\frac{1}{U_{i 0}^{\prime 2}+V_{i 0}^{\prime 2}}\left(U_{i 0}^{\prime}, V_{i 0}^{\prime}, 0\right)^{T}, \quad i=1,2
$$



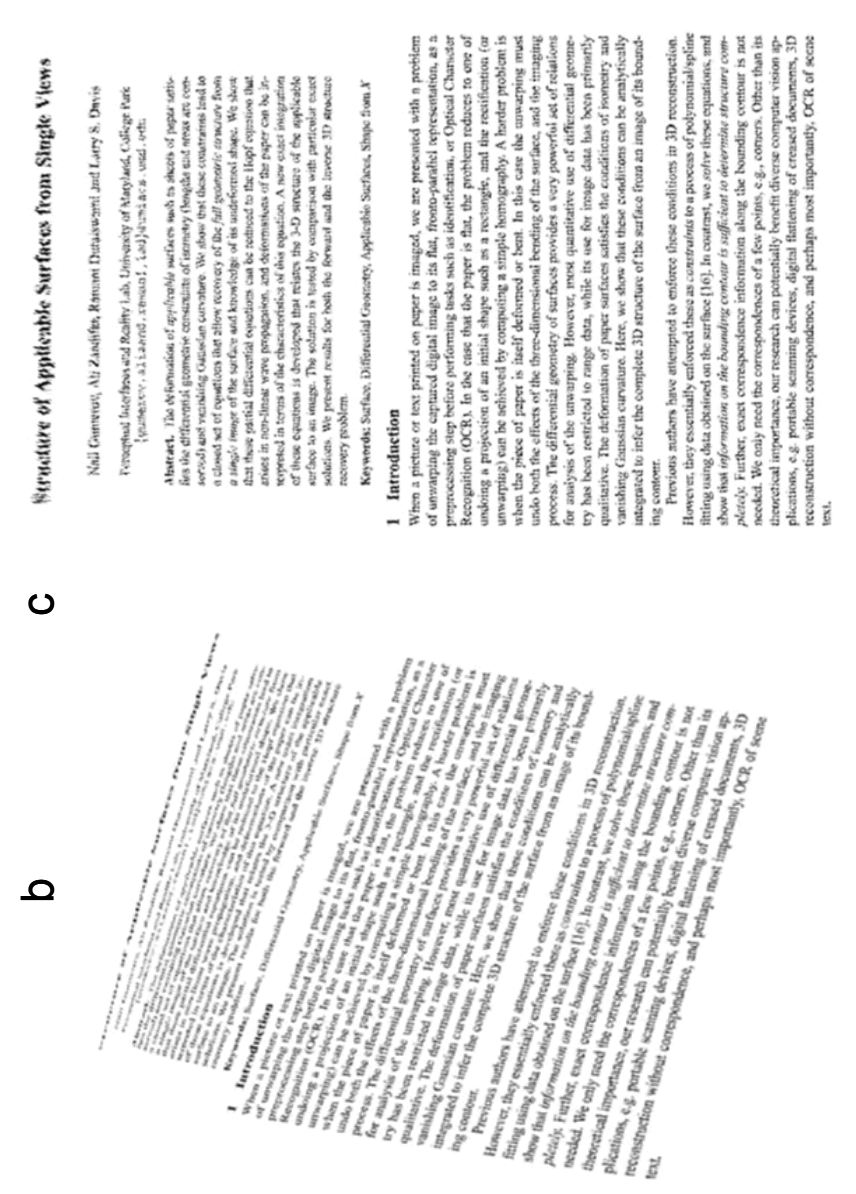

ఠ

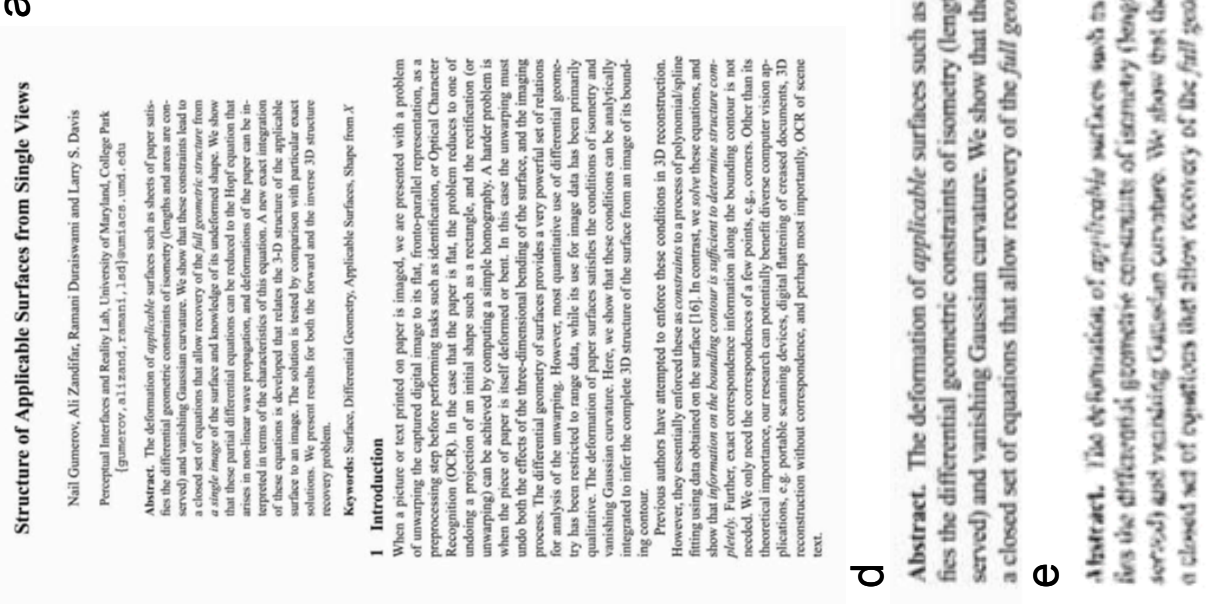

Figure 8: Inverse Problem for small font: a) original image; b) warped by the forward; c) unwarped by solution of the inverse problem; d) and f) zoom of the top and middle parts of a); e) and f) zoom of the same parts of the reconstructed (texture mapped) surface of c). 
Here we use superscript $T$ for transposition, to indicate that $\mathbf{j}_{i 0}(i=1,2)$ are column vectors. These vectors can also be written

$$
\mathbf{j}_{i 0}=\left(\cos \varphi_{i 0}, \sin \varphi_{i 0}, 0\right)^{T}, \quad i=1,2 .
$$

where $\varphi_{i 0}$ are the polar angles, which can be easily found from the given derivatives of the boundary curves $U_{i 0}^{\prime}$ and $V_{i 0}^{\prime}$.

Using equations (67), (79), and (94), we can rewrite the first equation (85) as

$$
\left(Q_{0} \mathbf{j}_{i 0}\right) \cdot \mathbf{b}_{i 0}=0, \quad i=1,2
$$

Let us represent vector $\mathbf{b}_{i 0}$ using spherical polar coordinates $\left(\left|\mathbf{b}_{i 0}\right|, \chi_{i 0}, \psi_{i 0}\right)$ :

$$
\mathbf{b}_{i 0}=\left|\mathbf{b}_{i 0}\right|\left(\cos \psi_{i 0} \sin \chi_{i 0}, \sin \psi_{i 0} \sin \chi_{i 0}, \cos \chi_{i 0}\right)^{T}, \quad i=1,2,
$$

and insert this relation into equation (96) together with expressions for $Q_{0}(80)$ and $\mathbf{j}_{i 0}(95)$. The result can be written as:

$$
\begin{aligned}
& \cos \left(\alpha_{0}+\varphi_{10}\right)\left[\cos \left(\psi_{10}-\gamma_{0}\right) \sin \chi_{10} \cos \beta_{0}-\cos \chi_{10} \sin \beta_{0}\right]+ \\
& \sin \left(\alpha_{0}+\varphi_{10}\right) \sin \left(\psi_{10}-\gamma_{0}\right) \sin \chi_{10} \\
& \cos \left(\alpha_{0}+\varphi_{20}\right)\left[\cos \left(\psi_{20}-\gamma_{0}\right) \sin \chi_{20} \cos \beta_{0}-\cos \chi_{20} \sin \beta_{0}\right]+=0 . \\
& \sin \left(\alpha_{0}+\varphi_{20}\right) \sin \left(\psi_{20}-\gamma_{0}\right) \sin \chi_{20}
\end{aligned}
$$

To solve the system, one can express, e.g., $\alpha_{0}$ via $\gamma_{0}$ and $\beta_{0}$ as

$$
\alpha_{0}=-\varphi_{10}-\arctan \left[\frac{\cos \left(\psi_{10}-\gamma_{0}\right) \cos \beta_{0}-\cot \chi_{10} \sin \beta_{0}}{\sin \left(\psi_{10}-\gamma_{0}\right)}\right]+\pi n,
$$

where $n=0, \pm 1, \ldots$, and $-a \pi<\alpha_{0} \leqslant \pi$ and obtain an equation for $\beta_{0}$

$$
\begin{aligned}
& \varphi_{10}-\varphi_{20}+\arctan \left[\frac{\cos \left(\psi_{20}-\gamma_{0}\right) \cos \beta_{0}-\cot \chi_{20} \sin \beta_{0}}{\sin \left(\psi_{20}-\gamma_{0}\right)}\right] \\
& -\arctan \left[\frac{\cos \left(\psi_{10}-\gamma_{0}\right) \cos \beta_{0}-\cot \chi_{10} \sin \beta_{0}}{\sin \left(\psi_{10}-\gamma_{0}\right)}\right]=0,
\end{aligned}
$$

which follows from the second equation (98). This equation can be solved efficiently using standard iterative solvers. The solution specifies $\beta_{0}$ as a function of $\gamma_{0}$, and enables one to determine $\alpha_{0}$ from equation (99) as function of $\gamma_{0}$.

We note that equations (99) may have several roots, and so multiple solutions should be considered for a given $\gamma_{0}$. Some solutions may be non-feasible, while some may exhibit the nonuniqueness (caused by ambiguities) of the inverse problem solution. This can be easily illustrated for the case of orthographic projection, for which we have $\chi_{10}=\chi_{20}=\pi / 2$. In this case, $\beta$ only enters equations (98) as $\cos \beta$, so if some $\beta$ is a solution then so is its negative, $-\beta$. Furthermore, in this case, the angles $\alpha$ and $\gamma$ enter equations as arguments of $\pi$-periodic functions (such as $\tan \left(\alpha+\varphi_{0}\right)$ ). Thus, if the equations are valid for some $(\alpha, \beta, \gamma)$ they are valid for $(\alpha+\pi n, \pm \beta, \gamma+\pi m)$ as soon as $n, m$, and the sign of $\beta$ are selected in such a way that all the Euler angles lie in the range $(-\pi, \pi]$. We note that in the case of orthographic projection we can expect that to any valid solution $\mathbf{r}(u, v)=(X, Y, Z)$ will correspond some "mirror" solution $\mathbf{r}^{*}(u, v)=(X, Y,-Z)$, since we are trying to uncover the $3 \mathrm{D}$ structure from its $2 \mathrm{D}$ projection, which is the same for $\mathbf{r}(u, v)$ and $\mathbf{r}^{*}(u, v)$. This non-uniqueness corresponds to the fact that correct solutions exist for at least two different sets of the Euler angles $\left(\alpha_{0}, \beta_{0}, \gamma_{0}\right)$. 


\section{References}

[1] Brown M.S. and Seales W.B., Document restoration using 3D shape: A general deskewing algorithm for arbitrarily warped documents In International Conference on Computer Vision, ICCV 2001, 2001.

[2] Clark P. and Mirmehdi M. (2001) Estimating the orientation and recovery of text planes in a single image In: Proceedings of the British Machine Vision Conference.

[3] Do Cormo M., Differential Geometry of Curves and Surfaces. Prentice Hall, 1976.

[4] Faugeras O., Three Dimensional Computer Vision. MIT press, 1990.

[5] Gray A., Modern Diffrential Geomtery of curves and surfaces with MATHEMATICA In CRC Press, 1998.

[6] Gumerov N. , Zandifar A., Duraiswami R. and Davis L.S. (2004) Structure of Applicable Surfaces from Single Views European Conference on Computer Vision (ECCV2004).

[7] Kanungo T., Haralick R., and Phillips I., Nonlinear Local and Global Document Degradation Models In Int'l. Journal of Imaging Systems and Technology, Vol. 5, No. 4, pp 220-230, Sep 1994.

[8] Kergosien Y.L., Gotoda H. and Kunii T.L., Bending and creasing virtual paper In IEEE Computer graphics and applications, Vol. 14, No. 1, pp 40-48, Jan 1994.

[9] Koenderink J.J., What Does the Occluding Contour Tell us About Solid Shape? Perception, 13: 321-330, 1984.

[10] Koenderink J.J., Solid Shape, MIT Press, 1990.

[11] Korn G.A. and Korn T.M., Mathematical Handbook for scientists and engineers In Dover Publications, Inc., 1968.

[12] Liebowitz D. and Zisserman A. (1998) Metric Rectification for Perspective Images of Planes In: IEEE Computer Vision and Pattern Recognition Conference. pp 482-488

[13] McInerney T. and Terzopoulos D., Deformable Models in Medical Image Analysis: A Survey, Medical Image Analysis, 1(2), 1996, pp 91-108.

[14] McIvor A. M., Robust 3D Surface Property Estimation In Second Asian Conference on Computer Vision, Vol. 2, pp 275-279, Dec 1995.

[15] Penna M.A., Non-rigid Motion Analysis: Isometric Motion In CVGIP: Image Understanding, Vol. 56, No. 3, pp 366-380, Nov 1992.

[16] Pilu M., Extraction of illusory linear clues in perspectively skewed documents In IEEE Computer Vision and Pattern Recognition Conference, Dec 2001. 
[17] Pilu M., Undoing Page Curl Distortion Using Applicable Surfaces In Proc. IEEE Conf Comouter Vision Pattern Recognition, Dec 2001.

[18] Press W.A., Teukolsky S.A., Vetterling W.T. and Flannery B. P., Numerical Recipes in C, Cambridge University Press, 1993.

[19] Wang Y.F., Mitchie A. and Aggarwal J.K., Computation of surface orientation and structure of objects using grid coding In IEEE trans. on pattren analysis and machine intelligence, Vol. 9, No. 1, pp 129-137, Jan 1987.

[20] Whitham G.B., Linear and Nonlinear Waves, New-York: Wiley, 1974.

[21] You Y., Lee J. and Chen Ch., Determining location and orientation of a labeled cylinder using point pair estimation algorithm In International Journal of Pattern Recognition and Artificial Intelligence, Vol. 8, No. 1, pp 351-371, 1994.

[22] Zandifar A., Chahine A., Duraiswami R. and Davis L.S. (2002) Video-based Interface to Textual Information for the Visually Impaired In: IEEE Computer Society, Internation Conference on Multimodal Interfaces (ICMI). pp 325-330. 